\title{
Microcosmic Mechanism of Die-sinking Mixed-gas Atomization Discharge Ablation Process on Titanium Alloy
}

\section{Linglei Kong}

Jiangsu University of Technology

\section{Xiangzhi Wang}

Changzhou Institute of Technology

\section{Qing He}

Jiangsu University of Technology

\section{Jinjin Han}

Jiangsu University of Technology

\section{Suorong Zhang}

Jiangsu University of Technology

\section{Kai Ding} Jiangsu University of Technology

Zhidong Liu ( $\sim$ liutim@nuaa.edu.cn )

Nanjing University of Aeronautics and Astronautics

\section{Research Article}

Keywords: Titanium alloy, EDM, Discharge ablation process, Mixed-gasatomized medium, Single pulse,Microcosmicmechanism

Posted Date: May 7th, 2021

DOl: https://doi.org/10.21203/rs.3.rs-486811/v1

License: (a) (1) This work is licensed under a Creative Commons Attribution 4.0 International License. Read Full License

Version of Record: A version of this preprint was published at The International Journal of Advanced Manufacturing Technology on August 3rd, 2021. See the published version at https://doi.org/10.1007/s00170-021-07736-6. 


\title{
Microcosmic mechanism of Die-sinking Mixed-gas Atomization
}

\author{
Discharge Ablation Process on Titanium alloy \\ Linglei Kong ${ }^{1,2}$, Xiangzhi Wang ${ }^{3}$, Qing He ${ }^{1}$, Jinjin Han $^{1}$, zhang Suorong ${ }^{1}$, Ding kai ${ }^{1}$, Zhidong \\ Liu*2 \\ (1. School of Mechanical Engineering, Jiangsu University of Technology, Changzhou, 213001, \\ China. 2. College of Mechanical and Electrical Engineering, Nanjing University of Aeronautics \\ and Astronautics, Nanjing 210016, China. 3. Jiangsu Key Laboratory of Non-traditional \\ Machining, Changzhou Institute of Technology, Changzhou 210032, China.)
}

\begin{abstract}
In order to reveal the microscopic mechanism of the titanium alloy die-sinking mixedgas atomization discharge ablation process (MA-DAP), a single-pulse-discharge test was performed using a needle electrode discharge device. According to the step-like characteristic of the single discharge waveform, the micro-erosion process could be divided into four stages: preparation for oxidation ablation; oxidation occurrence and oxide layer generation; oxidation-ablation dynamic balance and oxide layer prototype formation; oxidation-ablation termination and integral oxide layer formation. Theoretical analysis showed that the violent exothermic oxidation reaction between the oxygen medium and the high-temperature melting material introduced a large quantity of external chemical energy into the sparking region. Fast vaporization and explosion of the atomized medium after heating gave MA-DAP high particle removal power. The technological experimental results showed MA-DAP provides high material removal efficiency and strong deephole machining ability. The depth-to-diameter ratio of the specially shaped titanium alloy MA-DAF blind hole was 11 times that from traditional electrical discharge machining, while the crosssectional hardness can be reduced by $45 \%$.
\end{abstract}

Keywords: Titanium alloy; EDM; Discharge ablation process; Mixed-gas atomized medium; Single pulse; Microcosmic mechanism

\section{Introduction}

Titanium alloy materials with light weights, corrosion resistance, good biocompatibility, and other characteristics are widely used in aerospace, biomedicine, and other fields [1,2]. However, the high strength, high chemical activity, and low thermal conductivity of titanium alloy make it a typical difficult-to-machine material [3,4]. Electrical discharge machining (EDM) is a no-contact processing technology that is not affected by the hardness or thermal properties of the material to be machined, and is thus suitable for processing of titanium alloy materials $[5,6]$. However, the machining efficiency of EDM is lower than that of conventional mechanical cutting [7,8]. Moreover, EDM uses hydrocarbon-based dielectrics during machining. This produces hazardous gases and creates environmental and operator health and safety issues $[9,10]$.

Dry EDM is a new process variant of EDM that uses gas as the dielectric. It is friendly to the machining environment and offers high machining accuracy and low electrode loss [11-13]. In-

\footnotetext{
* Corresponding author.

E-mail address: liutim@nuaa.edu.cn

Tel: +86-13770600084
} 
depth studies of dry EDM have identified problems such as low processing efficiency and shortcircuit machining [14,15]. In order to improve the shortcomings of dry EDM, the near-dry EDM method was developed using two-phase, gas-liquid media and has attracted the attention of and deep research from experts and scholars [16-18]. The literature search found that both dry and near-dry EDM research have focused on processing of steel materials rather than difficult-to-machine materials such as titanium alloys or high temperature alloys and that the energy used to remove material is delivered using pulsed power supplies [12-20]. For this reason, Kong et al. [21,22] proposed a titanium-alloy mixed-gas discharge ablation processing method based on dischargeinduced ablation processing technology $[23,24]$ with oxygen as the main component and nitrogen or argon as auxiliary gases. The chemical energy generated by the reaction between oxygen and the titanium alloy greatly improved the rate of titanium alloy. Gas media such as nitrogen or argon with controllable oxygen concentrations were used to achieve high efficiency and stability throughout the titanium alloy processing sequence. However, this machining method is similar to dry EDM, which has a high machining short circuit rate and provides poor surface quality. In this paper, a titanium alloy die-sinking mixed-gas atomization discharge ablation process (MA-DAP) method is proposed. The mixture gas and working fluid are mixed at high pressure to form an atomization medium. The atomization medium is used to improve the ablation processing state between the electrodes in order to improve the ablation formability of the titanium alloy.

Various researchers have used needle-shaped electrodes and plate-shaped workpieces to enable point-board, single-pulse-discharge-mode systems in order to explore EDM mechanisms. Tani et al. [25] observed a workpiece being processed using a single discharge system via a high-speed camera equipped with a high-magnification lens. They found that single-discharge material removal occurred not only once, but several times under discharge conditions in which the pulse duration exceeded tens of microseconds. Govindan P. et al. [26] studied the monopulse discharge mechanism of magnetic field assisted dry EDM using needle electrodes. The experimental results showed that magnetic field assistance increased the machining depth and reduced the crater diameter by $80 \%$ during dry EDM. Zhang et al. [27] studied the material characteristics of various dielectric materials in air; oxygen; liquid dielectrics; de-ionized water; kerosene; and water in oil media via singlepulse-discharge with a needle electrode. Shen et al. [28] found that the workpiece can receive more energy from a needle electrode discharge than from a cylindrical electrode. F. T. B. Macedo et al. [29] studied the mechanism of single-pulse electrospark ablation in various gas media using a needle electrode. F. Klocke et al. [30] found that a large volume of material is removed when the titanium alloy is connected to the positive electrode.

In order to further improve the machining effect of mixed-gas discharge ablation processing of titanium alloy, based on the previous research results [21,22], the MA-DAP technology is proposed. Firstly, this paper introduces the principle of the MA-DAP technology. Then, the needle-plate singlepulse-discharge-mode systems was constructed and is used to study the material erosion miromechanism of the proposed technology. Based on the waveform characteristics of single pulse discharge, the change of inter electrode discharge channel and the mechanism of material erosion were analyzed. Finally, the continuous pulse spark discharge experiment is used to verify the efficiency and high-quality etching mechanism of the MA-DAP of titanium alloys.

\section{The principle of MA-DAP and the experimental platform}

\subsection{The principle of MA-DAP}


Fig. 1 illustrates the basic principle of MGADAF. A high concentration of oxygen and a relatively low concentration of nitrogen were mixed to form a mixed gas. The mixed gas enters the atomizing device together with water at a certain pressure to form an atomized medium as the machining dielectric for the ablation process. The oxygen component participates in the oxidation reaction and ablation process to provide external energy for the erosion and continuous oxidation of the workpiece material. The nitrogen component in the dielectric could restrict the extent of oxidation by controlling the diffusion rate of the oxygen to reduce the thickness of the recast layer.The high flow of the dielectric is beneficial for the removal of erosion products. All the above factors together achieve the stability and sustainability of the new processing method [21].

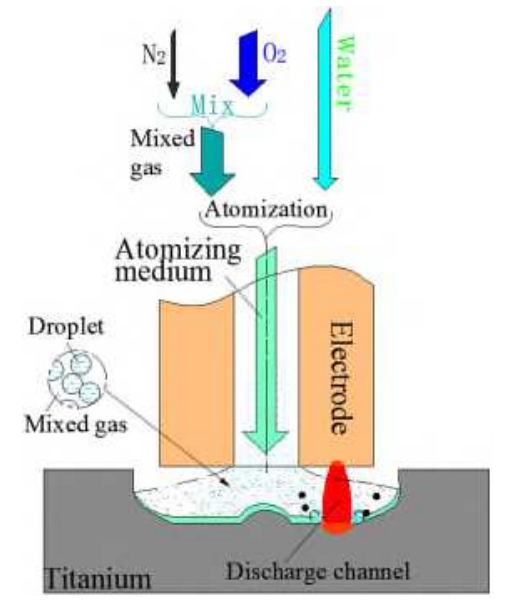

Fig. 1. Schematic diagram of MA-DAP.

\subsection{The experimental platform}

This study used a needle electrode to study the MA-DAP material ablation mechanism. The principle of the single-pulse-discharge test device is shown in Fig. 2. It consisted primarily of a workbench; electrode-motion control module; discharge-detection module; DC power supply and chopper; electrode-clamping device; hollow auxiliary copper tube and needle electrode; and an oscilloscope for voltage and current acquisition.

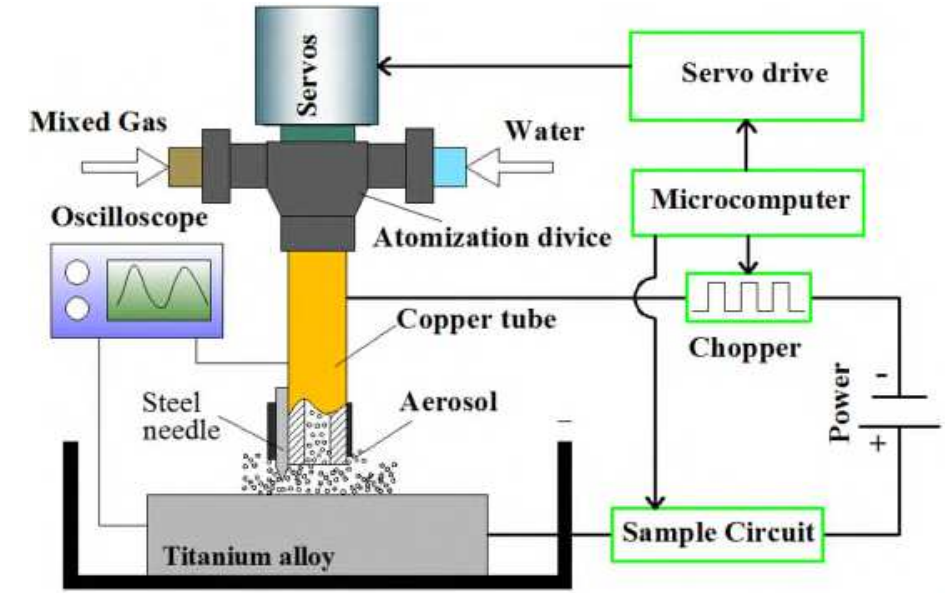

Fig. 2. Principle of the single-pulse-discharge test device with a needle electrode.

The single-pulse-discharge device used a lab-made DC power supply that was connected to the electrode and workpiece via the chopper. The chopper controlled power supply switching and pulse widths, while also modulating the pulsed power supply. The control system consisted of servo-drive 
and discharge-detection modules. In the experiment, the single-chip microcomputer controlled the electrode as it approached the workpiece at $1 \mu \mathrm{m}$ per step and judged whether discharge was occurring by detecting whether there is a change in the current between the two electrodes. If there was no discharge between the electrodes, the needle electrode remained near the workpiece. If there was discharge between the electrodes, the single-chip microcomputer retrieved the needle electrode. The single-pulse-discharge device is shown in Fig. 3. Single-pulse-discharge experimental parameters are shown in Table 1.

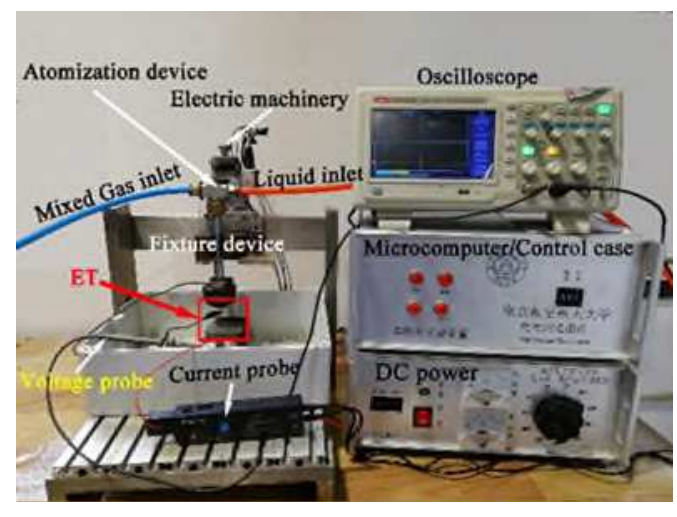

(a) Photograph of the test equipment

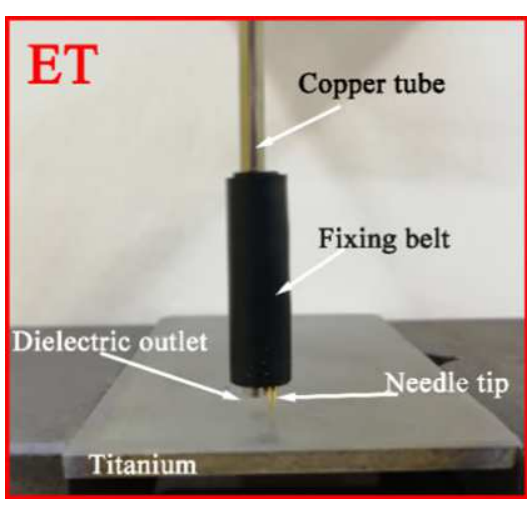

(b) a picture showing needle-plate discharge.

Fig. 3. Needle electrode single-pulse-discharge test device.

Table 1. Single-pulse-discharge experimental parameters

\begin{tabular}{cc}
\hline Parameters & Values \\
\hline Voltage / V & 250 \\
Pulse duration / $\mu \mathrm{s}$ & 600 \\
Mixed gas $\left(\mathrm{O}_{2}: \mathrm{N}_{2}\right)$ & $6: 1$ (Gas pressure ratio $)$ \\
Atomization quantity / $\mathrm{ml} / \mathrm{min}$ & 40 \\
Mixed gas pressure / MPa & 0.3 \\
Tool electrode $($ Steel needle & \\
material $)$ & Steel $45^{\#}(1045$, American standard $)$ \\
Workpiece & Ti-6Al-4V \\
\hline
\end{tabular}

To reveal the discharge mechanism and observe the discharge phenomenon, the single pulse discharge experiments should be carried out in the presence of dielectric fluid. To optimize the distance value $h$ between the needle tip and the outlet surface of the auxliary hollow electrode, the commercial CFD software FLUENT was emloyed to analyze the different flow field conditions of the machining with different $h$ values. Fig. 4 illustrates the geometric model. 


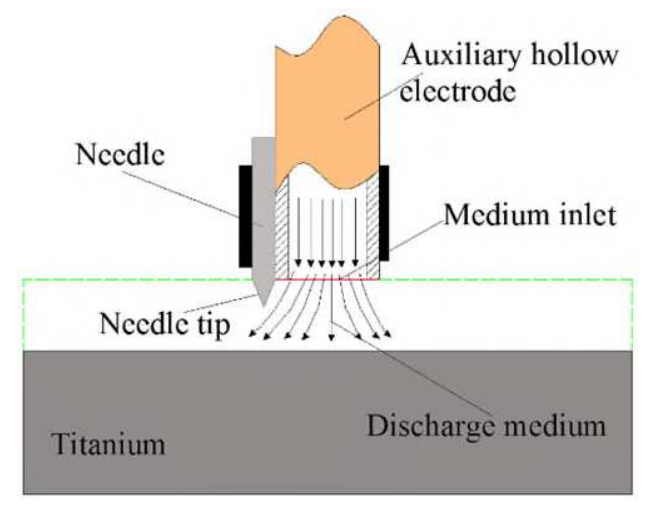

(a) The experimental principle

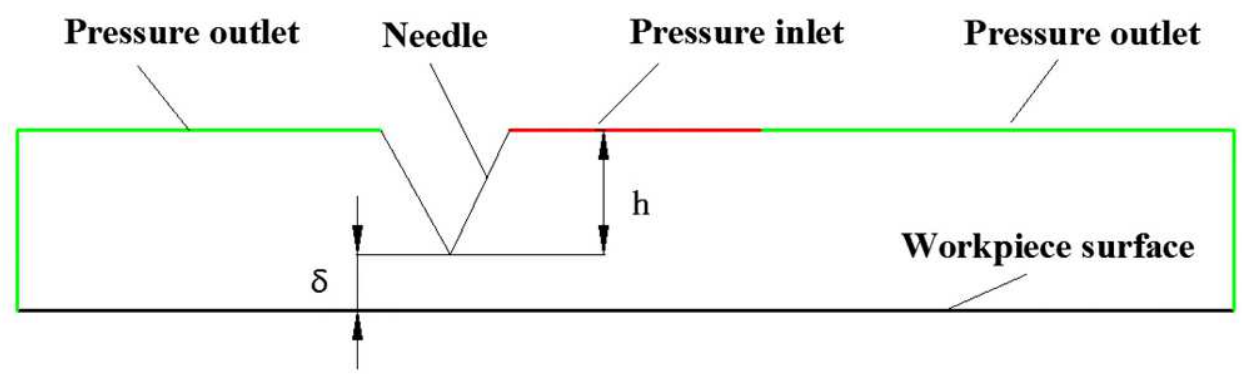

(b) The geometry model

Fig. 4 The construction of geometric simulation model of single pulse needle-plate

During the simulation process, the pressure inlet was set to the outlet of the auxiliary hollow electrode, the pressure outlet was set to the outsides of the electrodes. The inlet pressure was 0.3 MPa and the outlet pressure was environmental pressure. The sparking gap $\delta$ was fixed to investigate the influence of the difference value $h$ between the two electrodes on the flow field of the gap. The $\mathrm{k}-\varepsilon$ turbulent model was selected as the calculation model based on the Reynolds number (Re) [31]. Fig. 5 illustrates the different velocity nephogram calculated with different distances (difference value $h$ ).
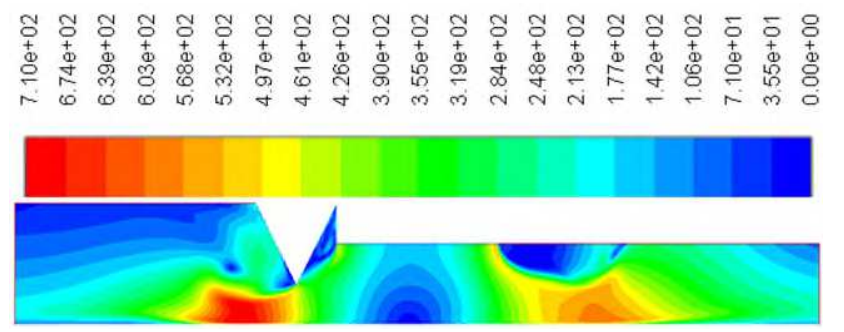

(a) $\mathrm{h}=0.5 \mathrm{~mm}$

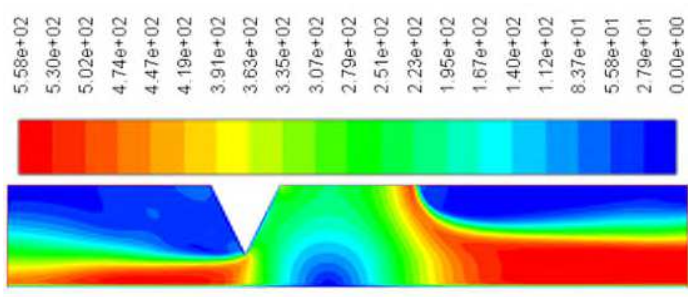

(b) $h=1 \mathrm{~mm}$

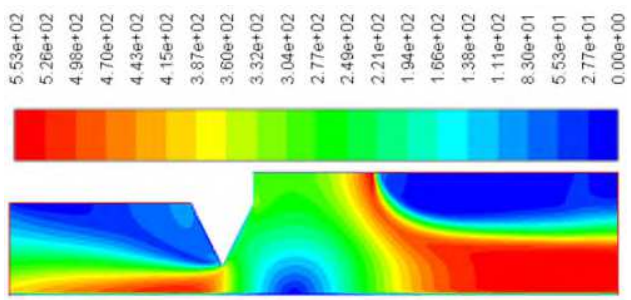

(c) $\mathrm{h}=1.5 \mathrm{~mm}$

Fig. 5 The different flow fields calculated with different distances.

It can be seen from Fig. 5 that the liquid flow velocity increases as the decrease of the distance $h$, resulting in a faster dielectric exchange rate, which is closer to the real discharge environment. The 
smaller the $\mathrm{h}$ is, the better the experimental results. However, due to the angle of the needle tip, it was difficult to set $0.5 \mathrm{~mm}$ to the distance $\mathrm{h}$ in the actual operation process, so the $\mathrm{h}=1 \mathrm{~mm}$ was an operational option.

\section{Micro-mechanism of MA-DAP}

The atomizing medium is a two-phase gas-liquid medium. When it is sprayed on the workpiece surface at high pressure, the force of the liquid-phase medium water supply, which has its own physical and chemical properties, forms a liquid film on the workpiece surface. The dielectric may have three states: gas, water droplets, and liquid film, as shown in Fig. 6.

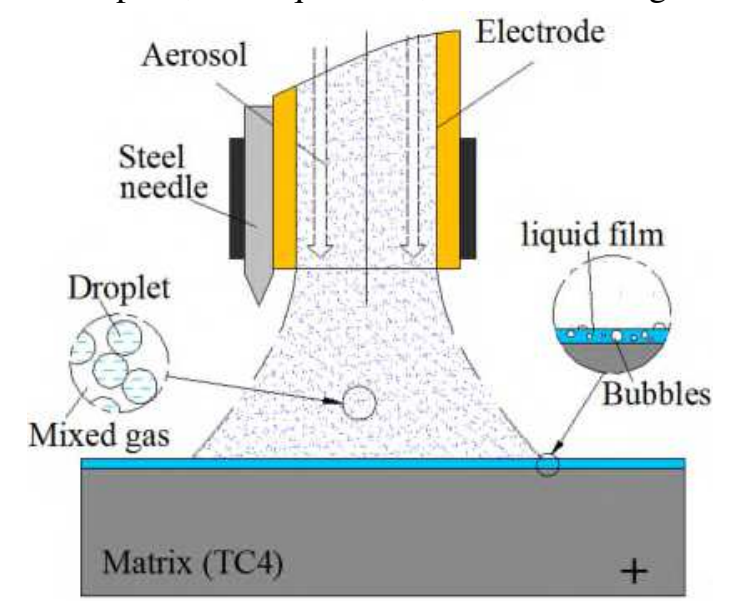

Fig. 6. Schematic diagram of the liquid film on the workpiece surface.

Fig. 7 shows single-pulse-discharge waveforms from MA-DAP and dry EDM. There is a significant difference between the waveforms. The former voltage discharge waveform exhibits an obvious step-like characteristic, which shows that the internal characteristics of the discharge channel have changed. Due to addition of oxygen to the MA-DAP system, an exothermic chemical reaction is included in the ablation process. Thus, the microscopic ablation mechanism is more complex than that of dry EDM. The micro ablation process can be divided into four stages based on the characteristics of the discharge voltage waveform.

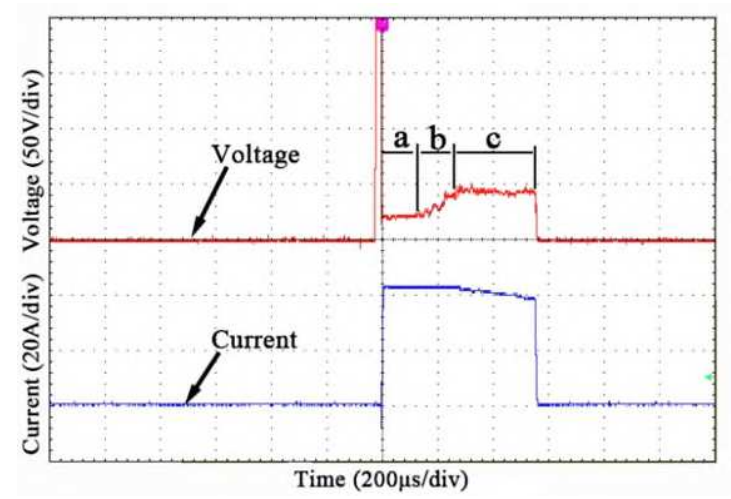

(a) MA-DAP

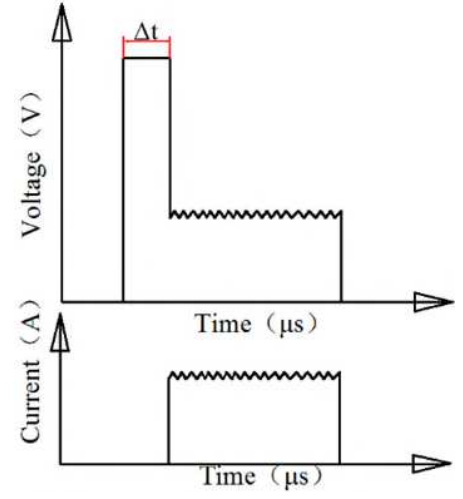

(b) Dry EDM [32]

Fig. 7. Single-pulse-discharge waveforms

\section{(1) Preparation for oxidation ablation}

This stage corresponds to Section 'a' shown in Fig. 7a. The dielectric in the sparking area will 
ionize and breakthrough under the action of the electric field formed by the electric voltage. The high-speed electrons will bombard the liquid film and workpiece surface, and form a plasma channel. The plasma channel will expand rapidly due to the high press in the channel. With the expansion of the discharge channel, the density of the internal charged particles decreases while the speed of movement accelerates. The discharge current rises, and the voltage decreases to the working voltage.The bombardment vaporizes part of the metal material and heats a lot of the material into the molten state, as shown in Fig. 8.

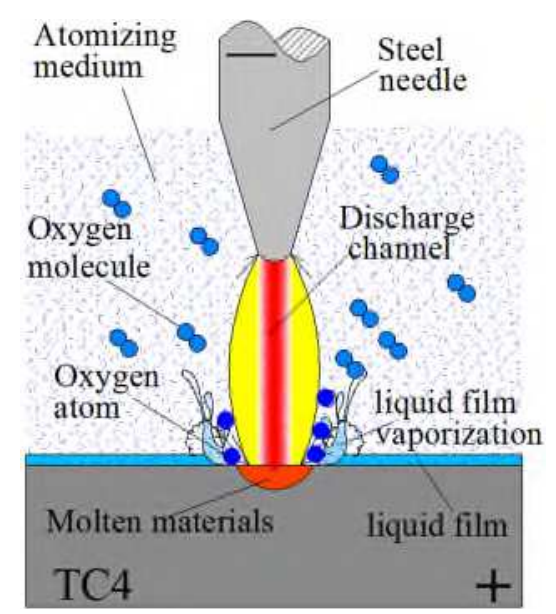

Fig. 8. The discharge channel and molten material.

It can be seen from Fig. 8 that the formation and expansion of the plasma channel has an effect on the decomposition of water in the dielectric. The electrolysis occurs when an electric voltage is applied to the sparking gap:

$$
\begin{gathered}
2 \mathrm{H}_{2} \mathrm{O} \rightarrow \mathrm{O}_{2}+4 \mathrm{H}^{+}+4 e^{-} \\
2 \mathrm{H}^{+}+2 e^{-} \rightarrow \mathrm{H}_{2}
\end{gathered}
$$

The liquid-phase dielectric (water) will decompose under the action of the high-temperature of the plasma channel:

$$
2 \mathrm{H}_{2} \mathrm{O} \stackrel{\text { high temperature }}{\longrightarrow} 2 \mathrm{H}_{2} \uparrow+\mathrm{O}_{2} \uparrow
$$

Thus, electrolysis of the water medium increases the quantity of electrons in the discharge channel, the probability of bombardment the positive electrode, and the transformation of kinetic energy to thermal energy in the discharge channel. The oxygen generated by the decomposition can enhance the effect of the oxidation reaction. To verify the ionization and decomposition of water in the atomizing medium under the influence of the discharge channel, a liquid water medium was used as the working medium for titanium alloy EDM. Fig. 9 illustrates the energy spectrum analysis of titanium alloy surface by EDM in pure water dielectric. It can be seen from the figure that there were oxide products formed on the machined surface and an oxidation reaction took place during the machining process. 


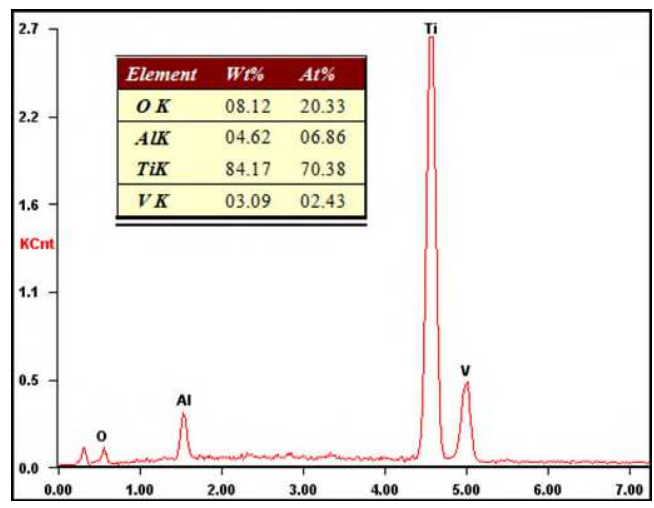

Fig. 9. Energy spectrum analysis of the titanium alloy surface after EDM in water

At high temperatures, oxygen molecules will undergo a series of chemical reactions, such as dissociation and ionization [33]. As shown in Fig. 10, the oxygen molecule in the dielectric can be ionized or dissociated from the ground state oxygen molecule to high energy state oxygen atoms or particles. These oxygen atoms and particles are more likely to participate in the oxidation reaction under the action of the plasma channel's high temperature and provide a combustion supporting medium for the next ablation stage.

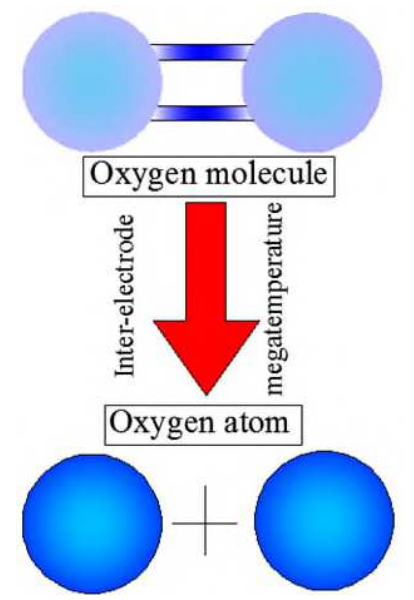

Fig. 10. Dissociation of oxygen molecules.

\section{(2) Oxidation-ablation occurrence and oxide layer generation}

This stage corresponds to section ' $b$ ' in Fig. 7(a). The increase of discharge voltage indicates a change in the inter-electrode discharge channel. This occurs because the high-temperature molten material and oxygen particles generated in the previous stage begin to undergo an oxidative ablation reaction that produces titanium oxide, which has a relatively high resistivity [21,22]. The occurrence of oxidation causes the internal pressure of the discharge channel to change. As a result, the electromagnetic binding force that it generates is insufficient to maintain its balance and the discharge channel diameter gradually expands. The expansion of the discharge channel causes the pressure on the surface of the workpiece material to decrease, thus allowing the high-pressure partial molten metal to be thrown away from the discharge pit as pressure is lost. The partially oxidized material remains in the discharge channel and induces oxide layer formation. In addition, the oxygen produced from liquid water may participate in the inter-electrode oxidation etching reaction. The microscopic mechanism by which this stage occurs is shown in Fig. 11. 


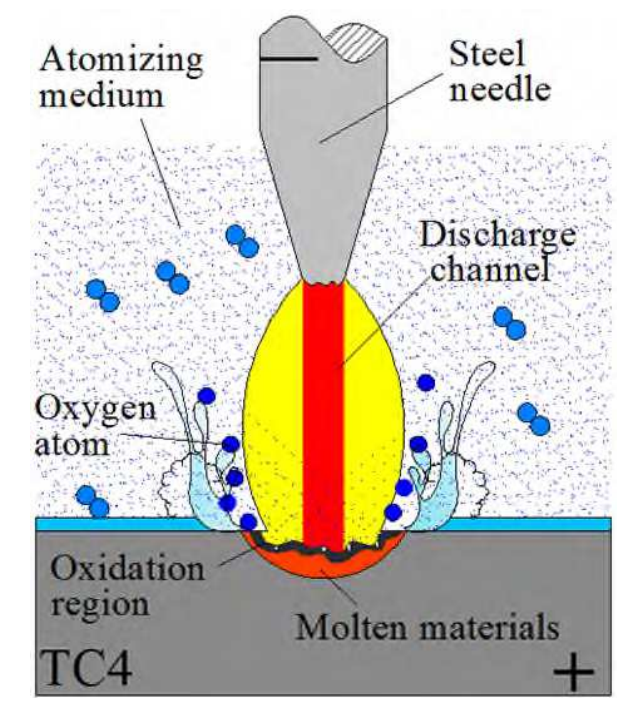

Fig. 11. Oxidation occurrence and oxide layer generation

Large amounts of thermal and chemical energy are generated via oxidation ablation and introduced into the inter-electrode. This aids in the efficient removal of titanium alloy materials. If one assumes that all of the inter-electrode titanium alloy oxidation reactions generate $\mathrm{TiO}_{2}$, the heat that can be released after the complete oxidation of $1 \mathrm{~mol}$ of titanium to $\mathrm{TiO}_{2}$ is $\mathrm{Q}_{\mathrm{R}}=9.44 \times 10^{5} \mathrm{~J} / \mathrm{mol}$. The heat $\mathrm{Q}_{\mathrm{A}}$ required to heat $1 \mathrm{~mol}$ of titanium alloy from room temperature $\left(\mathrm{T}_{1}=25{ }^{\circ} \mathrm{C}\right)$ to melting temperature $\left(\mathrm{T}_{2}=1668^{\circ} \mathrm{C}\right)$ is:

$$
Q_{A}=C m\left(T_{2}-T_{1}\right)=41075 \mathrm{~J} / \mathrm{mol}
$$

The latent heat of fusion of the titanium alloy is $\mathrm{Lm}=2.092 \times 10^{4} \mathrm{~J} / \mathrm{mol}$. Therefore, the heat released via oxidation of $1 \mathrm{~mol}$ of titanium alloy during ablation can melt $\mathrm{M}$ mole of the titanium alloy metal the melting point:

$$
M=\frac{1 * Q_{R}}{Q_{A}+L_{m}}=15.23 \mathrm{~mol}
$$

Therefore, the heat released after the complete oxidation of 1 mol of titanium alloy can heat 15.23 moles of titanium alloy to the molten state. It is obvious that introduction of the oxygen medium can provide a large amount of chemical energy between the electrodes and thus support efficient removal of titanium alloy material.

\section{(3) Oxidation-ablation dynamic balance and oxide layer prototype formation}

This stage corresponds to section 'c' of Fig. 7(a). Since the discharge voltage tends to balance, the resistance formed in the inter-electrode discharge channel tends to balance. That is, the dischargechannel oxide layer thickness is nearly stable. During EDM, the charged particles in the discharge channel continuously bombard the workpiece surface to achieve rapid material removal. Thus, the discharge channel still has an effect on material removal during this stage. This means that the entire etching process is governed by an oxidation ablation dynamic equilibrium in which the oxide layer is removed via discharge or oxidative stripping and simultaneously generated via oxidative etching of the lower layer titanium alloy material. Fig.12 illustrates the dynamic balance of oxidation ablation, this process is also the main stage of titanium alloy discharge ablation machining. It can be seen that the discharge voltage waveform will not change until the pulse duration ends, which shows that the oxide layer in the channel remains in the discharge pit until the discharge channel 
disappears. Therefore, the oxide layer is the main component of the final surface oxide layer, it is the embryonic form of the final oxide layer.

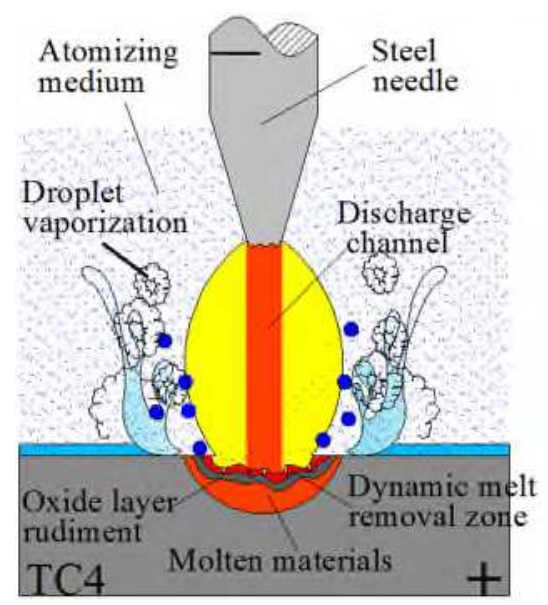

Fig. 12. Oxidation-ablation dynamic balance and oxide layer prototype formation

It can be seen from Fig. 10 that the liquid component formed by the water droplet and liquid film between the electrode and workpiece will rapidly vaporize under the high-temperature action of the plasma channel and the oxidative ablation reaction. The vaporization and expansion of the liquidphase component will form an explosive effect for the debris removal from the small sparking area. Studies have shown that when water at $100{ }^{\circ} \mathrm{C}$ is completely transformed into water vapor at, the volume change can be calculated using Eq. (6):

$$
V_{S}=\frac{1000 V_{l} k}{M}=\frac{1000 \times V_{l} \times 22.4}{18}=1244.44 V_{l}
$$

Where $V_{\mathrm{s}}$ is the volume of water vapor, $V_{l}$ is the volume of liquid water, $\mathrm{K}$ is the molar mass of the gas, and $\mathrm{M}$ is the molar mass of liquid water. Fig. 11 illustrates the inter-electrode model of the atomization ablation. To characterize the debris removal effect of the atomized dielectric at the moment of discharge, it is assumed that the volume of liquid in the sparking gap is half of the entire dielectric, then the volume of the vaporized fluid $\mathrm{V}_{\mathrm{a}}$ could be described as:

$$
V_{a}=1244.44(a+\delta)^{2} \delta / 2
$$

Assuming that the side gap is the same as the bottom discharge gap $(\delta)$, excluding the gas flow resistance, the height $h$ of the explosive eruption of water vapor that carries ablation products is:

$$
\mathrm{h}=1244.44(a+\delta)^{2} \delta / 2(a+\delta) \delta 4
$$

Substituting the adopted electrode side length $\mathrm{a}=5 \mathrm{~mm}$ into formula (8) produces $\mathrm{H}=785.6 \mathrm{~mm}$. Therefore, at the moment of discharge, explosion of the atomizing medium applies a large product discharge force. Fig. 14 shows the actual machining status of the MA-DAP. During machining, the titanium alloy combustion spark fills the entire discharge area and the ablation products are discharged in the form of an eruption. 


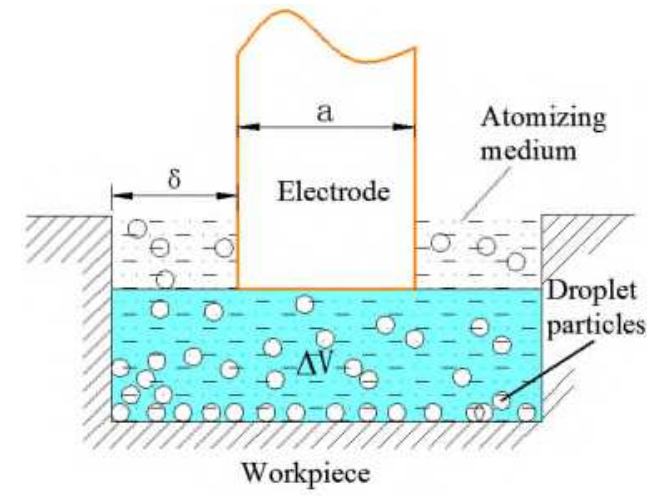

Fig. 13. A model of inter-electrode atomization ablation

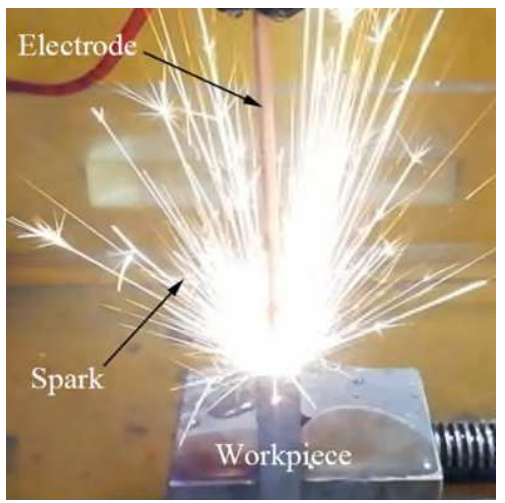

Fig. 14. The MA-DAP machining status.

\section{(4) Oxidation-ablation termination and integral oxide layer formation}

After the pulsed current terminates, deionization occurs between the electrodes and the discharge channel disappears, indicating the end of the entire discharge ablation process. This is accompanied by partially melted material being thrown out of the discharge ablation pit and inter-electrode cooling of the partially melted material and high-temperature oxide layer into solidified oxide. This forms an oxide layer on the titanium alloy sample surface, as shown in Fig. 15. The actual discharge ablation pit is shown in Fig. 16 and consists of two parts: the erosion pit and the oxidized molten ablation material. The energy spectrum of the discharge ablation pit surface (Fig. 17) shows that the oxygen contents of the etch pit (Fig. 17(a)) and the oxidized molten ablation material (Fig. 17(b)) are much higher than that of the titanium alloy matrix material. This indicates that the oxidation ablation reaction occurs during material erosion.

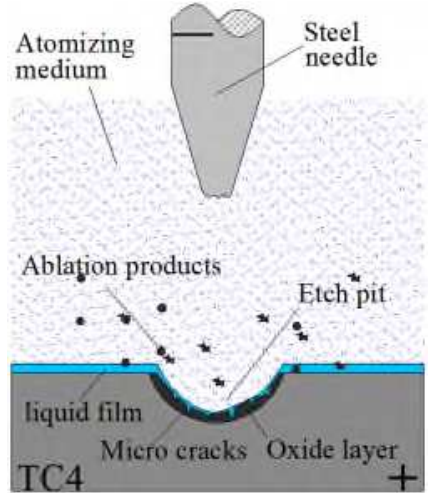

Fig. 13. The end of discharge ablation and oxide layer formation.

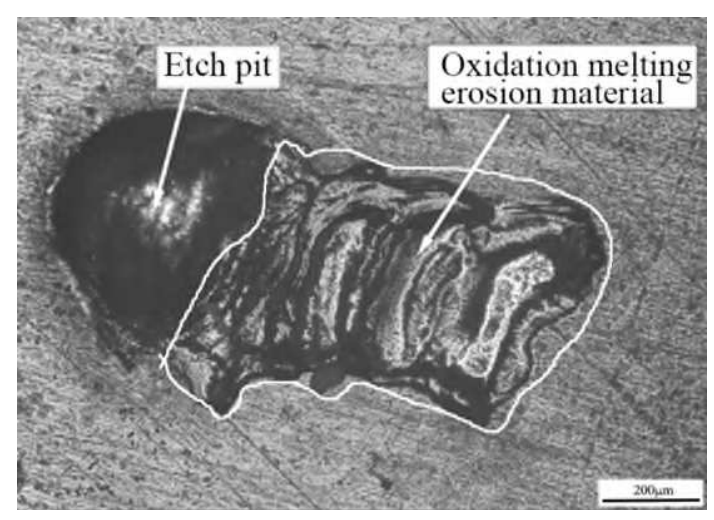

Fig. 14. A discharge ablation pit. 


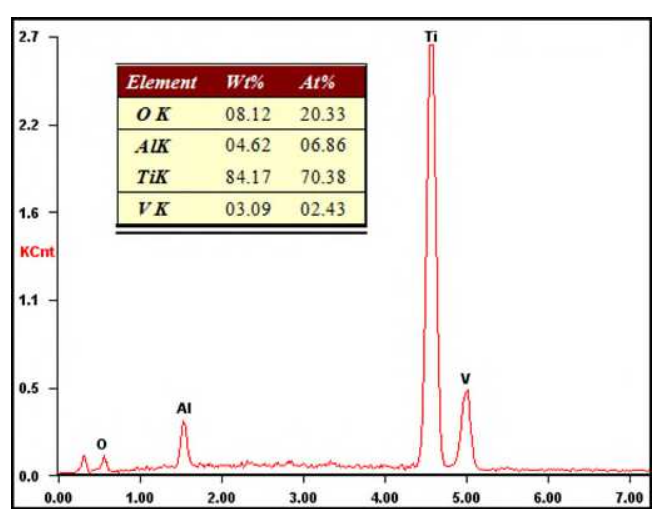

(a) Etch pit

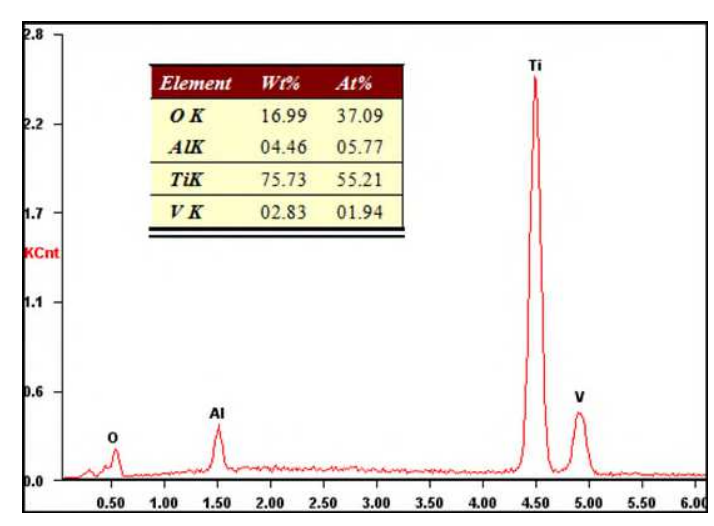

(b) oxidation melting erosion material.

Fig. 15. Discharge ablation pit surface energy spectrum detection

\section{Technological experiments of MA-DAP}

From the microscopic point of view, the use of oxygen in the mixed gas medium introduces a large amount of external chemical energy into the inter-electrode region, thus enabling efficient removal of titanium alloy materials. Furthermore, the explosive effect of rapid vaporization of the liquid medium in the atomizing medium gives MA-DAP strong particle removal capabilities. Therefore, in order to study the macroscopic characteristics of titanium alloy MA-DAP, a continuous pulse discharge experiment was performed and the results were compared to those from traditional EDM. The machining parameters used are shown in Table 2.

Table 2. Comparative processing experiment parameters

\begin{tabular}{ccc}
\hline Parameters & MA-DAP & EDM \\
\hline Current $(\mathrm{A})$ & 16 & 16 \\
Pulse duration/ Pulse interval $(\mu \mathrm{s})$ & $600 / 100$ & $600 / 100$ \\
$\mathrm{O}_{2}: \mathrm{N}_{2}($ Gas pressure ratio) & $6: 1$ & $\times$ \\
Gas Pressure $(\mathrm{MPa})$ & 0.4 & $\times$ \\
Atomization amount $(\mathrm{ml} / \mathrm{min})$ & $40($ water $)$ & $\times$ \\
Flushing pressure $(\mathrm{MPa})$ & $\times$ & $0.4(\mathrm{EDM}$ oil $)$ \\
Electrode size $(\mathrm{mm})$ & $5 \times 5$ & $5 \times 5$ \\
Workpiece material & \multicolumn{2}{c}{ Titanium alloy (TC4) }
\end{tabular}

Fig. 15 compares the MA-DAP and traditional EDM results. The MA-DAP depth and depth-todiameter ratio reach $56 \mathrm{~mm}$ and 11, respectively. Given the same processing time, the EDM depth is approximately $5.1 \mathrm{~mm}$ and the depth-to-diameter ratio is only 1 . This shows the efficiency of MA-DAP. In addition, during MA-DAP, the machining depth is linear with time (Fig. 15(b)). When the machining depth increases, the machining speed does not change substantially. This shows that the atomization medium vaporization expansion particle removal effect is good and has strong deephole machining capabilities. Photographs of workpieces machined using the two methods are shown in Fig. 15(a). These pictures show that although the MA-DAP depth is relatively large, the machining accuracy of the process is not lower than that of traditional EDM. 


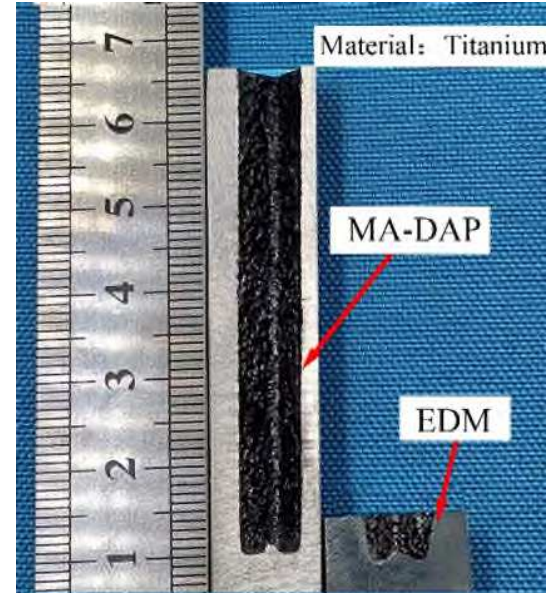

(a) Samples machined via EDM and

MA-DAP

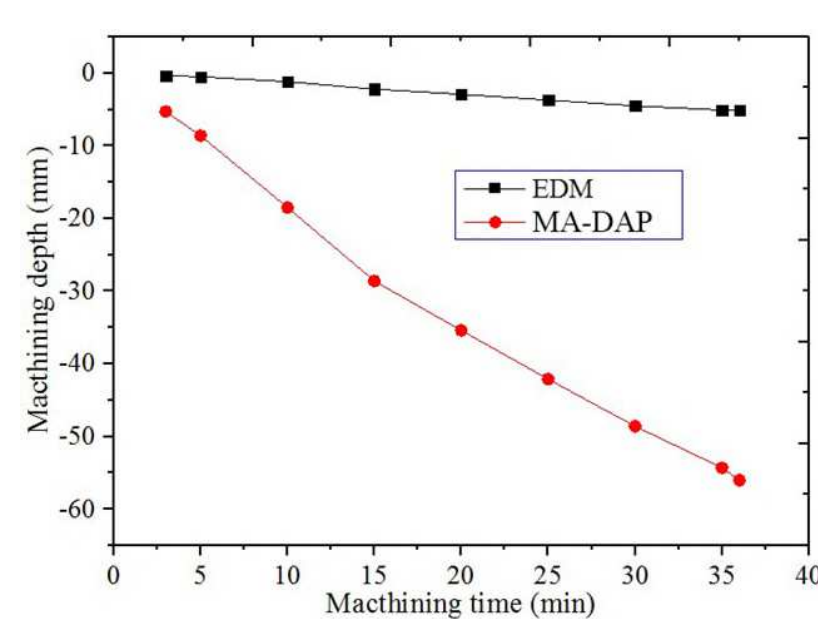

(b) The EDM and MA-DAP processes

Fig. 15 EDM and MA-DAP comparative test results

As shown in Figure $7 \mathrm{~b}$ the " $\Delta \mathrm{t}$ " is the breakdown delay time. The study shows that the larger $\Delta \mathrm{t}$ indicates the larger the discharge gap and good chip removal conditions; the smaller or zero $\Delta t$ indicates that the discharge gap is small and the worse the physical state of the inter-electrode medium deteriorates (the concentration of the etched products is too high or the local temperature is too high). At this moment, although each pulse is discharged in time, the productivity is low. Therefore, when etched products removal is difficult, it is beneficial to appropriately increase the breakdown delay time $\Delta$ t. Fig. 16 shows the discharge waveforms of the two processing methods. It can be seen that the number of normal discharge waveforms in MA-DAP is relatively large compared to EDM, and the breakdown delay time $\Delta \mathrm{t}$ is relatively wide, which indicates that the discharge gap increases under the effect of vaporization and explosion of liquid medium between electrodes, and the etched products removal condition between electrodes is good, which is beneficial to improve the machining efficiency of MA-DAP. It can also be seen from the figure that the number of abnormal discharge waveform and open circuit waveform of ma-dap is less due to the vaporization and explosion effect of inter electrodes liquid medium.

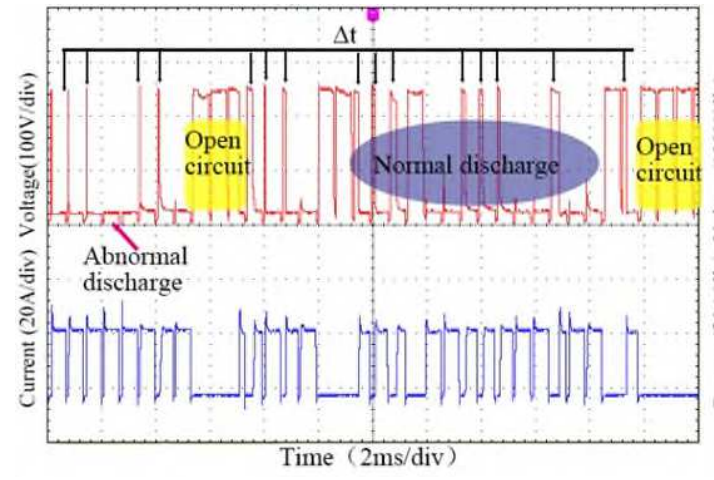

(a) MA-DAP

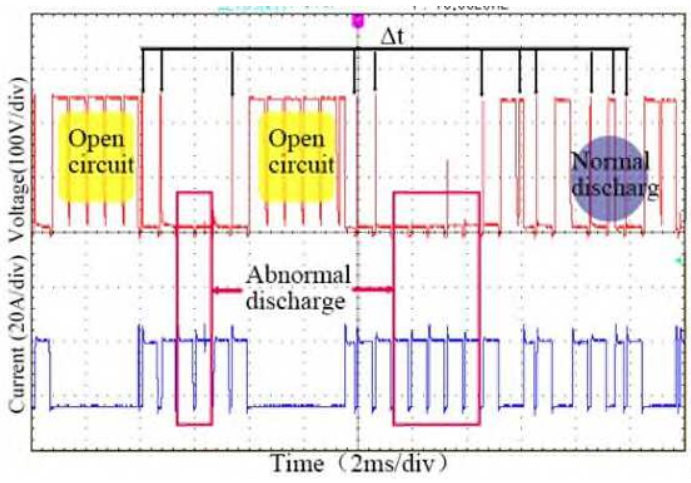

(b) EDM

Fig. 16. Comparison of discharge waveform

Fig. 17 compares cross-sectional hardness curves of titanium alloy workpieces processed via the two processing technologies. The MA-DAP surface hardness is much lower than that from EDM. There is a relative decrease of more than $45 \%$. At the same time, it can be seen from Fig. 17 that the thickness of modified layer of MA-DAP is also smaller than that of traditional EDM. This is because the products formed on the titanium alloy surface during MA-DAP are titanium alloy oxides. After 
traditional EDM, the surface consists mainly of titanium alloy carbide. This produces a harder surface. The reduction in the machined surface hardness means that subsequent workpiece processing requirements are correspondingly reduced. This is conducive to reducing the subsequent processing cost.

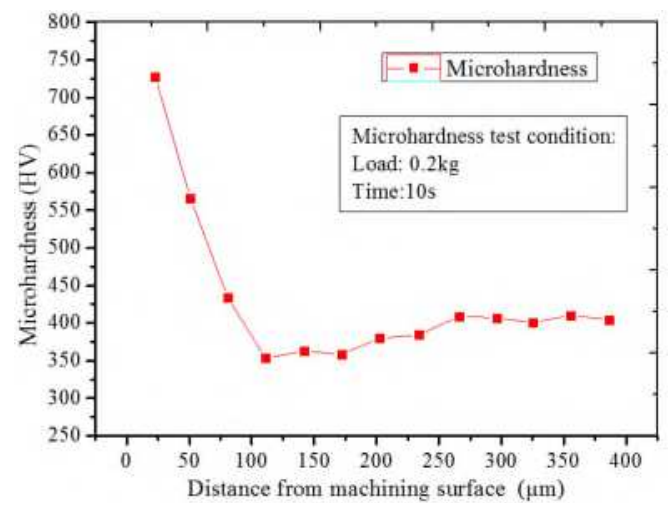

(a) MA-DAP

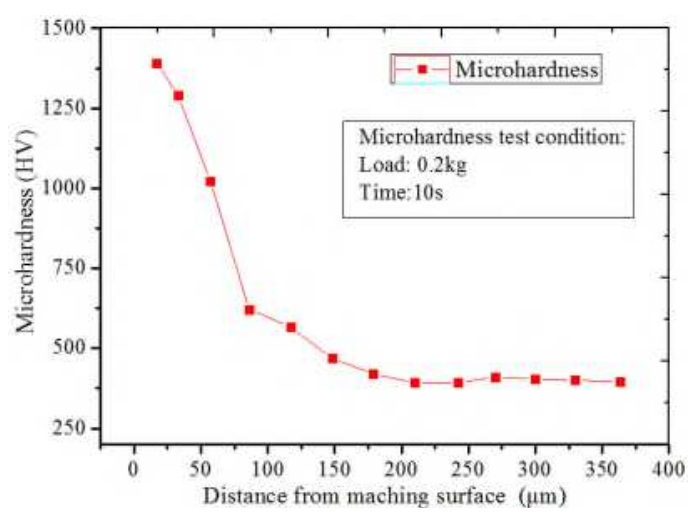

(b) EDM

Fig. 17. Cross-sectional hardness curves

\section{Conclusions}

This paper proposed a new MA-DAP for the machining of titanium alloy. According to the theoretical analysis and experimental investigation, the following conclusions can be obtained:

1. The typical single pulse discharge waveform exhibits a stepped characteristic due to the high resistivity of the oxide layer caused by the oxidation ablation reaction.

2. The micro-erosion process of MA-DAP could be divided into four stages: oxidationablation preparation, oxidation-ablation occurrence and oxide layer generation, oxidation-ablation dynamic balance and rudimentary oxide layer formation, oxidationablation termination and integral oxide layer formation.

3. The exothermic oxidation between oxygen and the high-temperature molten material induced by spark discharge provides a guarantee for the efficient processing of titanium alloys; the vaporization and explosion of the liquid-phase medium provide kinetic energy for expelling debris from the ablation area. This also gives it a wider breakdown delay, more normal discharge pulses and fewer abnormal discharge pulses than EDM.

4. Technological experiments proved that the depth-to-diameter ratio of a special-shaped blind hole in MA-DAP was more than 11 times higher than that of the traditional EDM, while the cross-sectional hardness can be reduced by $45 \%$. The mixed gas atomized dielectric had a stronger ability of vaporization expansion and particles removal, which is reflected in a linear relationship between the depth of the machined hole and time.

\section{Declarations}

Ethical approval Not applicable.

Consent to participate Not applicable.

Consent to publish Not applicable. 
Authors Contributions All authors have been personally and actively involved in substantive work leading to the report.

Funding This project was supported by the National Natural Science Foundation of China (Grant No. 51705040, 51975290). The authors extend their sincere thanks to those who contributed in the preparation of the instructions.

Competing Interests The authors declare that they have no competing of interest.

Availability of data and materials The data and materials set supporting the results are included within the article.

\section{References}

[1] Ezugwu EO, Bonney J, Yaman. An overview of the machinability of aero engine alloys. J. Mater. Process. Technol. 2003;134: 233-253.

[2] Yi S, Li S, Zhu S, Wang X, Mo J, Ding S. Investigation of machining Ti-6Al-4V with graphene oxide nanofluids: Tool wear, cutting forces and cutting vibration. J. Manuf. Proc. 2020;49: 3549.

[3] Yadav US, Yadava V. Experimental investigation on electrical discharge drilling of Ti-6Al-4V alloy. Mach. Sci. Technol. 2015;19: 515-535.

[4] Wang X, Liu Z, Xue R, Tian Z, Huang Y. Research on the influence of dielectric characteristics on the EDM of titanium alloy. Int. J. Adv. Manuf. Technol. 2014;72: 979-987.

[5] Gu L, Lei L, Zhao W, Rajurkar KP. Electrical discharge machining of Ti6Al4V with a bundled electrode. Int. J. Mach. Tools Manuf. 2012;53:100-106.

[6] Wang X, Li C, Guo H, Yi S, Kong L, Ding S. Alternating energy electrical discharge machining of titanium alloy using a WC-PCD electrode, J. Manuf. Proc. 2020;60:37-47.

[7] Sundaram MM, Pavalarajan GB, Rajurkar KP. A study on process parameters of ultrasonic assisted micro EDM based on Taguchi method, J. Mater. Eng. Perform. 2008;17: 210-215.

[8] Das S, Paul S, Doloi B. An experimental and computational study on the feasibility of biodielectrics for sustainable electrical discharge machining. J. Manuf. Process. 2019;41:284296.

[9] Valaki JB, Rathod PP, Khatri BC. Environmental impact, personnel health and operational safety aspects of electric discharge machining: a review. Proc. Inst. Mech. Eng., Part B: J. Eng. Manuf. 2015;229:1481-1491.

[10] Leão FN, Pashby IR. A review on the use of environmentally-friendly dielectric fluids in electrical discharge machining. J. Mater. Process. Technol. 2004;149:341-346. doi:10.1016/j.jmatprotec.2003.10.043.

[11] ZhanBo Y, Takahashi J, Kunieda M. Dry electrical discharge machining of cemented carbide. J. Mater. Process. Technol. 2004;149:353-357. doi:10.1016/j.jmatprotec.2003.10.044.

[12] Shen Y, Liu Y, Sun W, Zhang Y, Dong H, Zheng C, Ji R. High-speed near dry electrical discharge machining. J. Mater. Process. Technol. 2016;233:9-18. doi:10.1016/j.jmatprotec.2016.02.008

[13] Linglei K, Zhidong L, Mingbo Q, Wenzhao W, Han Y, Bai S. Machining characteristics of submersed gas-flushing electrical discharge machining of Ti6Al4V alloy. J. Manuf. Proc. 2019;41:188-196. 
[14] Govindan P, Joshi SS. Experimental characterization of material removal in dry electrical discharge drilling. Int. J. Mach. Tools. Manuf. 2010;50:431-443. doi:10.1016/j.ijmachtools.2010.02.004

[15] Macedo FTB, Wiessner M, Hollenstein C, Paulo MB, Wegener K. Fundamental investigation of dry electrical discharge machining (DEDM) by optical emission spectroscopy and its numerical interpretation. Int. J. Adv. Manuf. Technol. 2017;90: 3697-3709.

[16] Gholipoor A, Baseri H, Shakeri M, Shabgard M. Investigation of the effects of magnetic field on near-dry electrical discharge machining performance. Proc. Inst. Mech. Eng., Part B: J. Eng. Manuf. 2014;1-8.

[17] Krishnakant D, Akshay D. Parametric evaluation on near-dry electric discharge machining, Mater. Manuf. Processes. 2016;31(4):413-421.

[18] Vineet KY, Pradeep K, Akshay D. Effect of tool rotation in near-dry EDM process on machining characteristics of HSS, Mater. Manuf. Proc. 2019;34:779-790.

[19] Kumar, N. E. Arun, Babu, A. Suresh. Influence of input parameters on the near-dry WEDM of Monel alloy. Mater. Manuf. Proc. 2018;33: 85-92.

[20] Bai X, Zhang Q-H, Yang T-Y, Zhang J-H. Research on material removal rate of powder mixed near dry electrical discharge machining. Int. J. Adv. Manuf. Technol. 2013;68:17571766.

[21] Linglei K, Liu Z, Han Y, Qiu M. Research on the efficient and stable die-sinking electrical discharge machining ablation process of Ti-6Al-4V. Int. J. Adv. Manuf. Technol. 2017;80:704711.

[22] Linglei K, Zhidong L, Mingbo Q, Yunxiao H. Processing factors of controllable die-diesinking ablation processing on Ti-6Al-4V. J. Manuf. Proc. 2019;39:338-345.

[23] Zhidong L, An'yang X, Mingbo Q, Xu J, Li W. Basic study of combustion-material removal process by multi-function electrode. Procedia CIRP 2013;6:64-70.

[24] Liu Z, Wang X, Cao Z. Influence of discharge energy on EDM ablation. Int. J. Adv. Manuf. Technol. 2016;83:681-688.

[25] Takayuki T, Tsujita Y, Gotoh H, Okada M, Mohri N. Observation of material removal process by single discharge in air gap. Procedia CIRP 2018;68: 276-279.

[26] Govindan P, Gupta A, Joshi SS, Malshe A, Rajurkar KP. Single-spark analysis of removal phenomenon in magnetic field assisted dry EDM. J. Mater Proc. Tech. 2013;213:1048-1058.

[27] Zhang Y, Liu Y, Shen Y, Ji R, Li Z, Zheng C. Investigation on the influence of the dielectrics on the material removal characteristics of EDM. J. Mater. Process. Tech. 2014;214: 1052-1061.

[28] Shen Y, Liu Y, Zhang Y, Tan B, Ji R, Cai B, Zheng Ch. Determining the energy distribution during electric discharge machining of Ti-6Al-4V. Int. J. Adv. Manuf. Technol. 2014;70:1117.

[29] Macedo FTB, Wiessner M, Hollenstein C, Kuster F, Wegener K. Dependence of crater formation in Dry EDM on electrical breakdown mechanism. Procedia -CIRP 2016;42: 161166.

[30] Klocke F, Mohammadnejad M, Holsten M, Ehle L, Zeis M, Klink A. Comparative study of 
polarity-related effects in single discharge EDM of titanium and iron alloys. Procedia CIRP 2018;68:52-57.

[31] W. Koenig, R. Weill, R. Wertheim, W. I. Julzler. The flow fields in the working gap with electro discharge machining, Procedia CIRP 1977; 25 (1): 71-76.

[32] Takayuki T, Tsujita Y, Gotoh H, Okada M, Mohri N. Observation of material removal process by single discharge in air gap. Procedia CIRP 2018;68:276-279.

[33] Binggang D, Xiangyan K, Guohua D. Aerodynamics. Beijing: Higher Education Press; 1990 (in Chinese). 
Figures

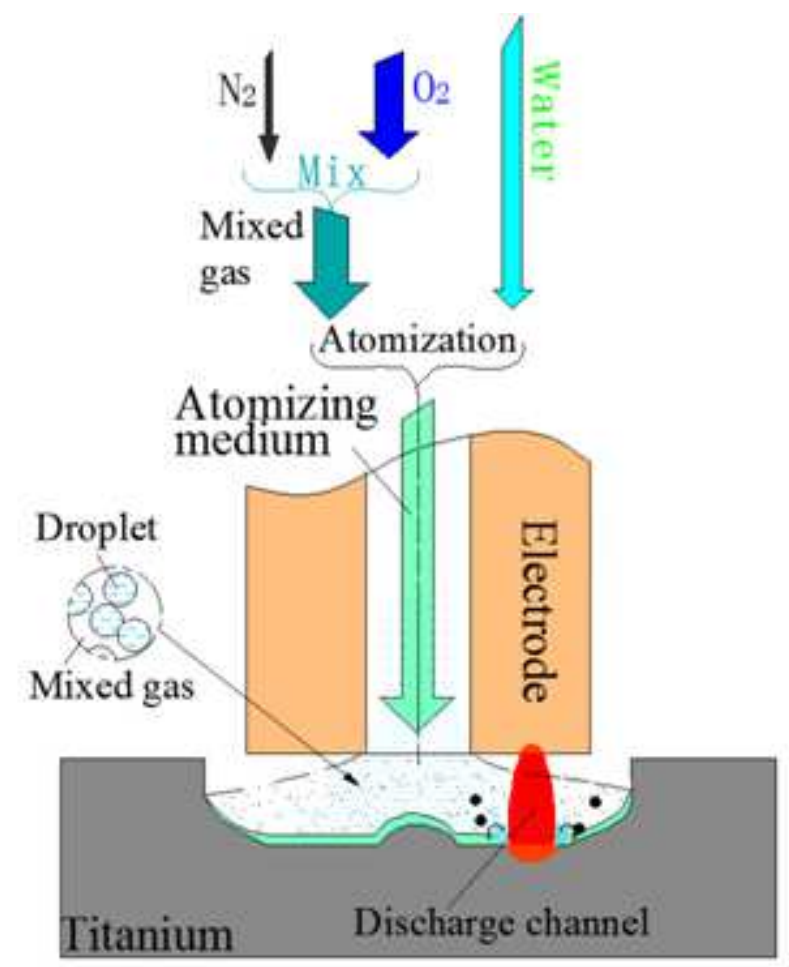

Figure 1

Schematic diagram ofMA-DAP.

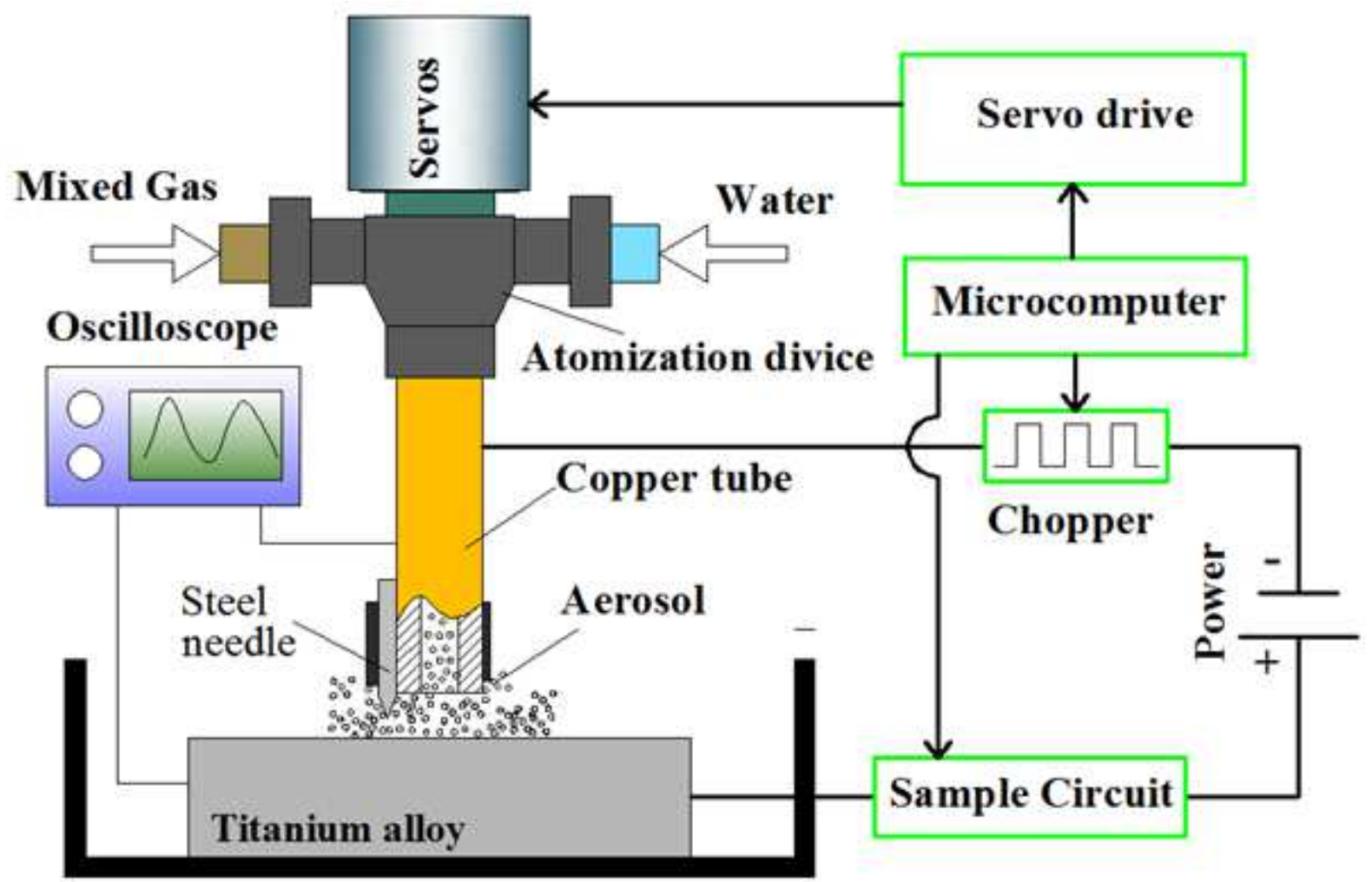

Figure 2 
Principle of the single-pulse-discharge test device with a needle electrode.

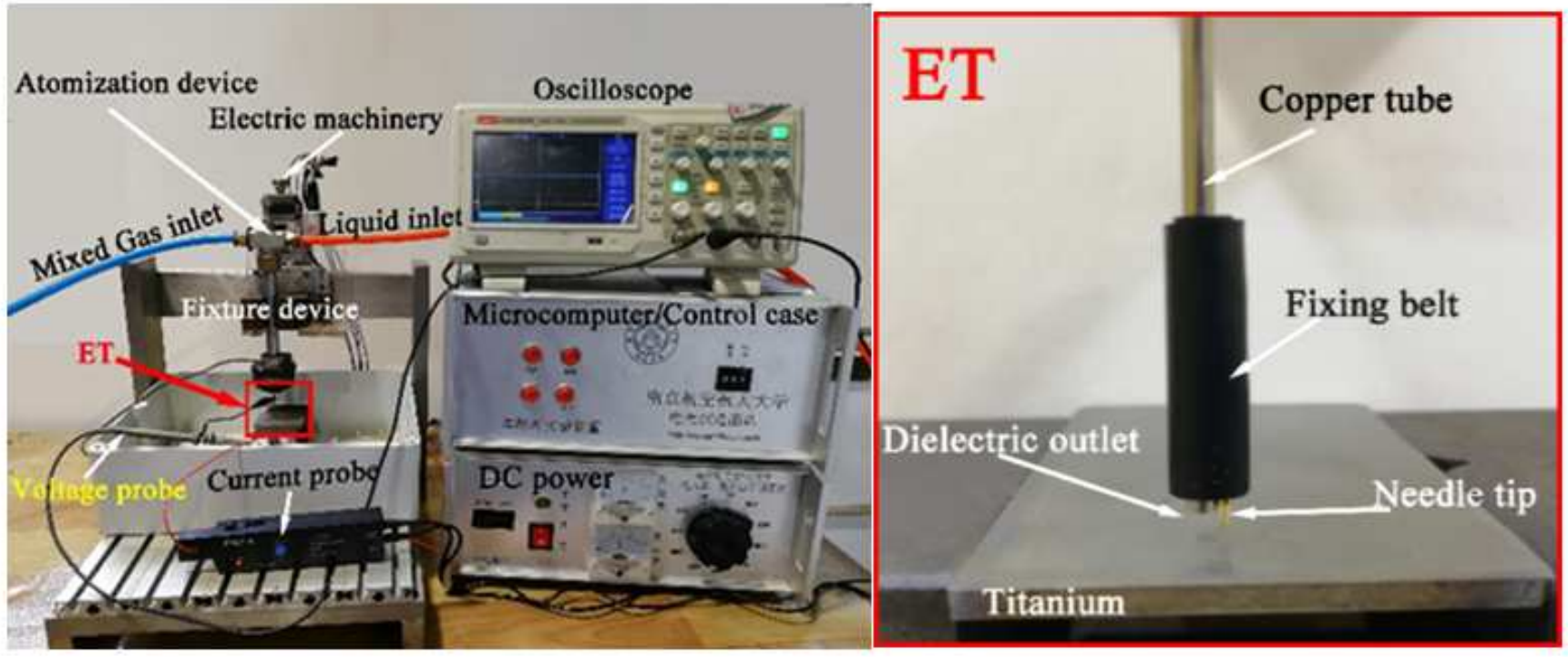

(a) Photograph of the test equipment

(b) a picture showing needle-plate discharge.

Figure 3

Needle electrode single-pulse-discharge test device.

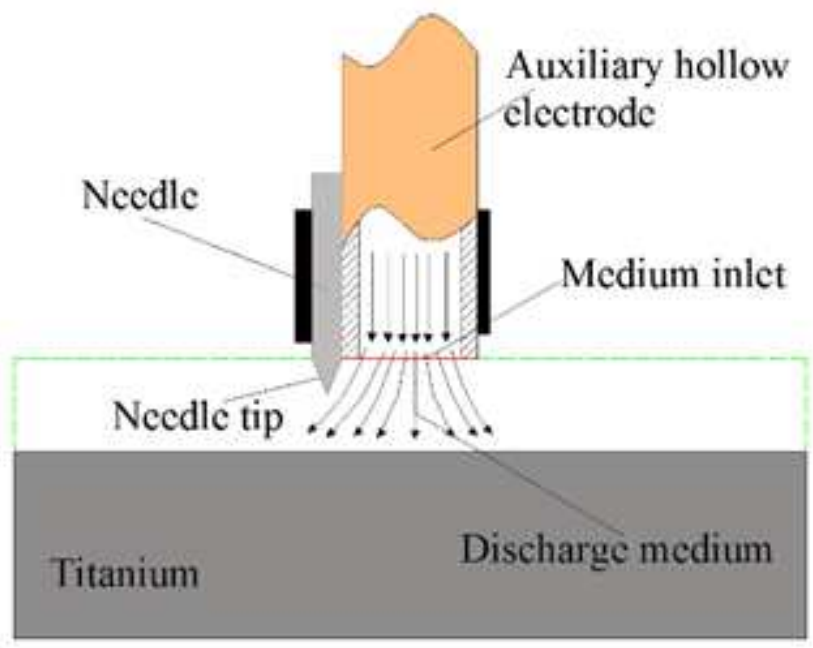

(a) The experimental principle

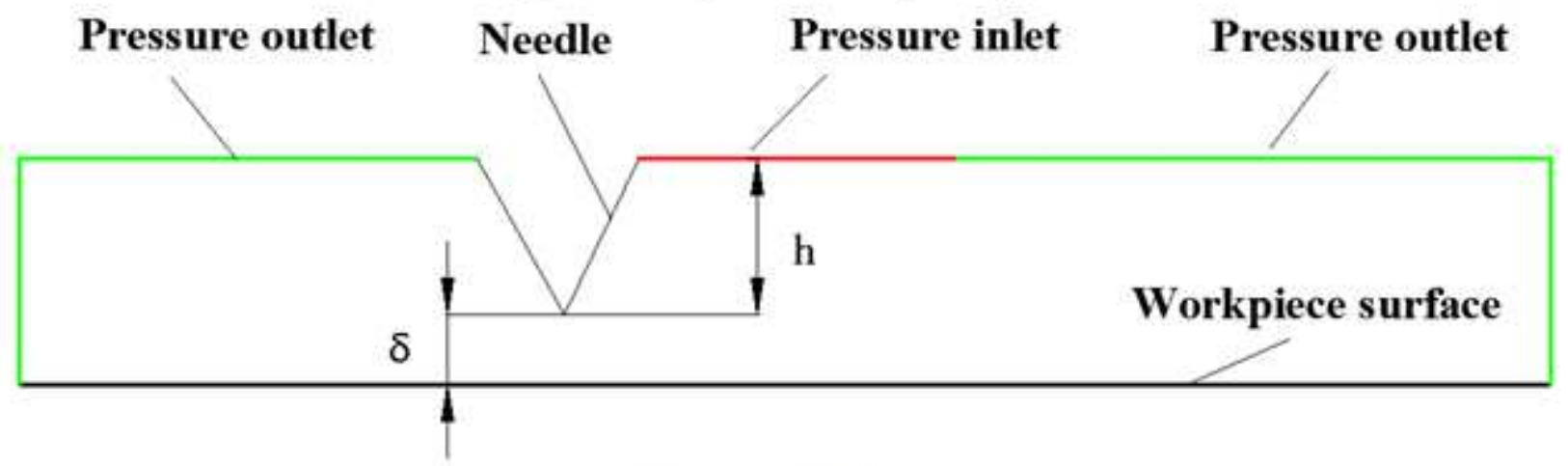

(b) The geometry model 
Figure 4

The construction of geometric simulation model of single pulse needle-plate
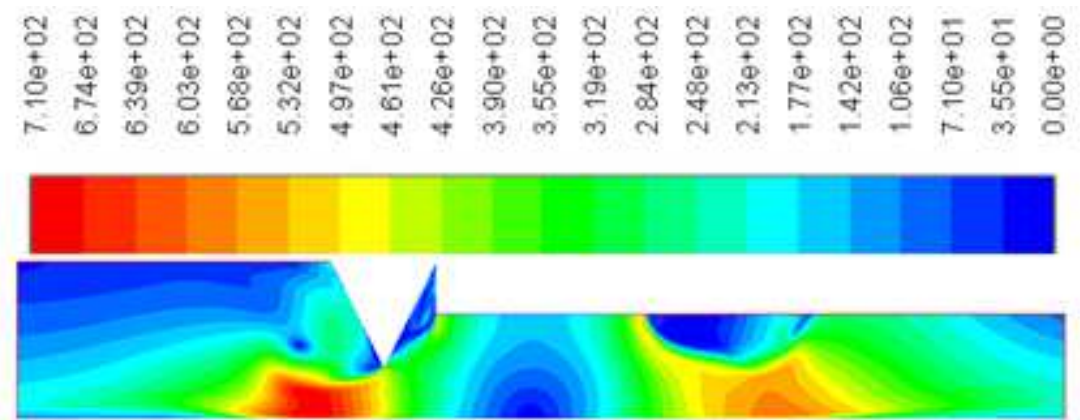

(a) $\mathrm{h}=0.5 \mathrm{~mm}$

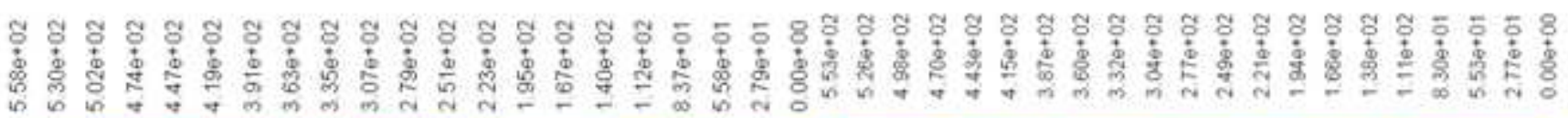

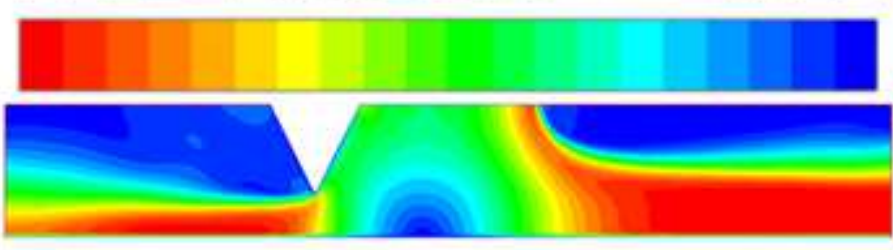

(b) $\mathrm{h}=1 \mathrm{~mm}$

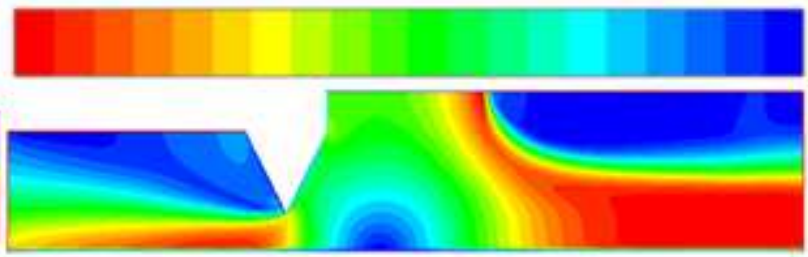

(c) $\mathrm{h}=1.5 \mathrm{~mm}$

\section{Figure 5}

The different flow fields calculated with different distances.

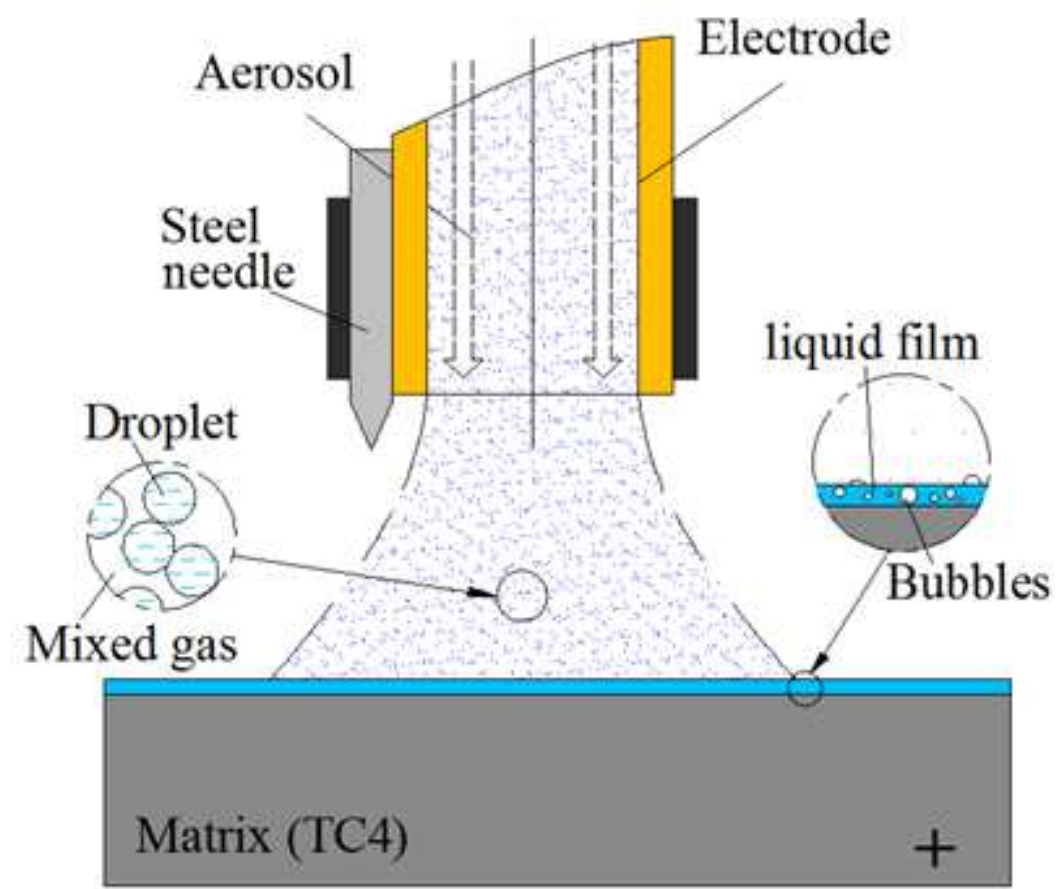

Figure 6 
Schematic diagram of the liquid film on the workpiece surface.

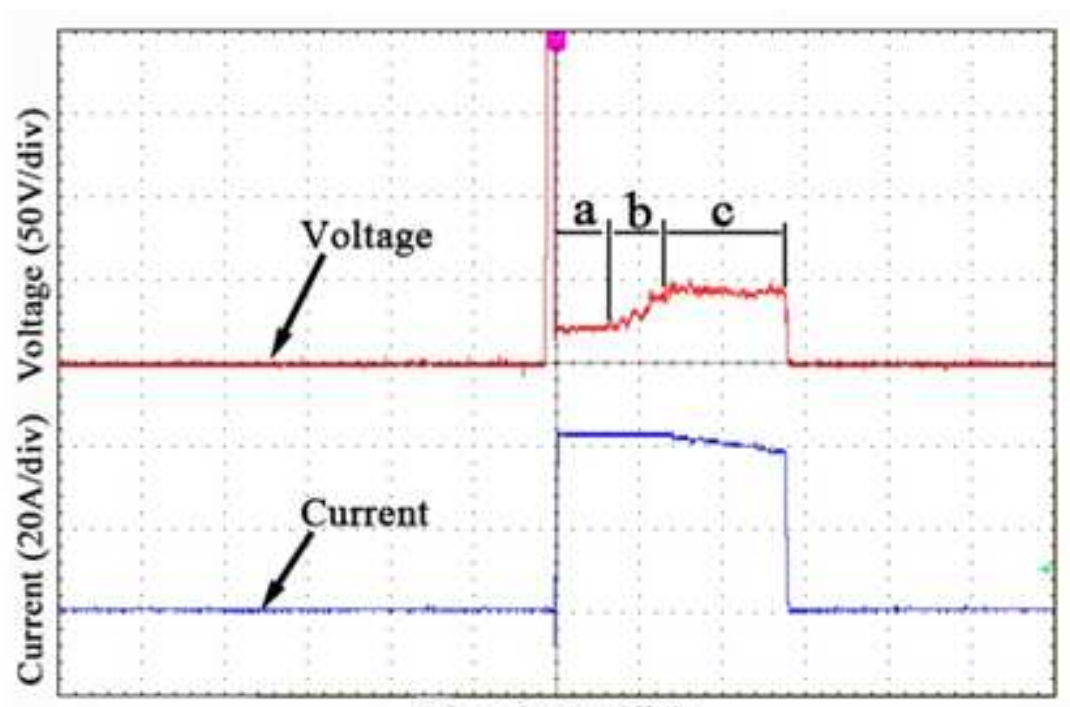

Time $(200 \mu \mathrm{s} /$ div $)$

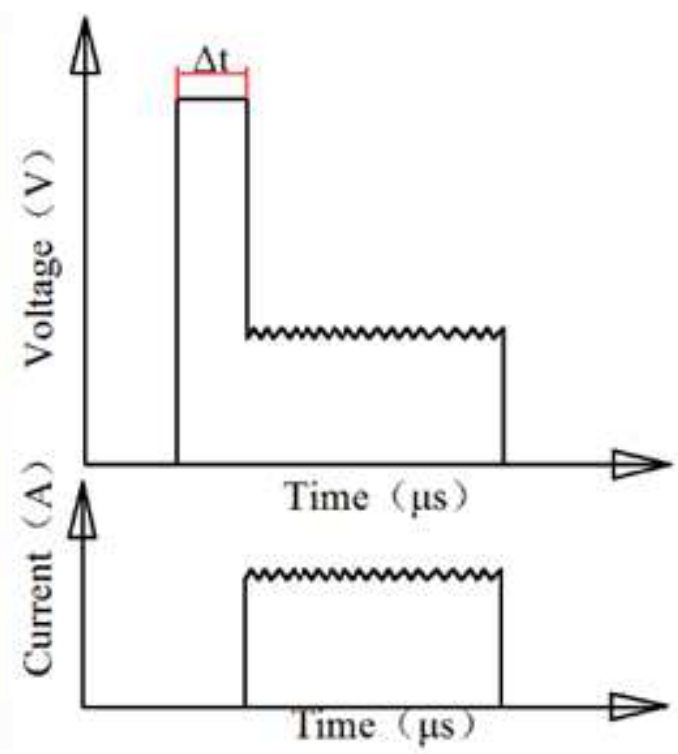

(a) MA-DAP(b) Dry EDM [32]

Figure 7

Single-pulse-discharge waveforms

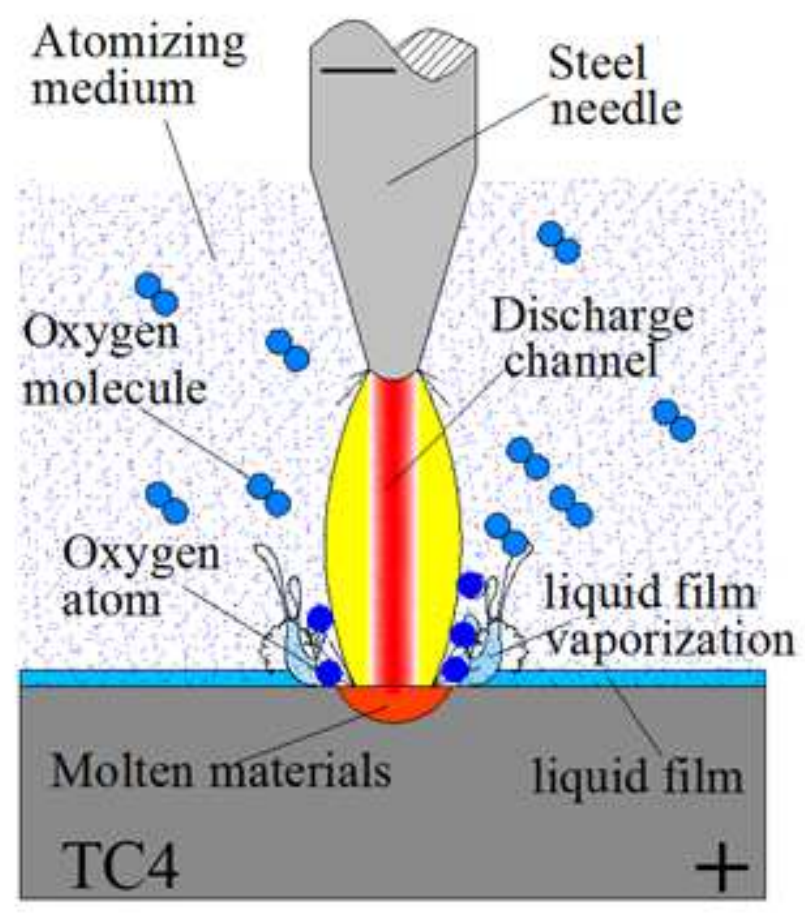

Figure 8

The discharge channel and molten material. 


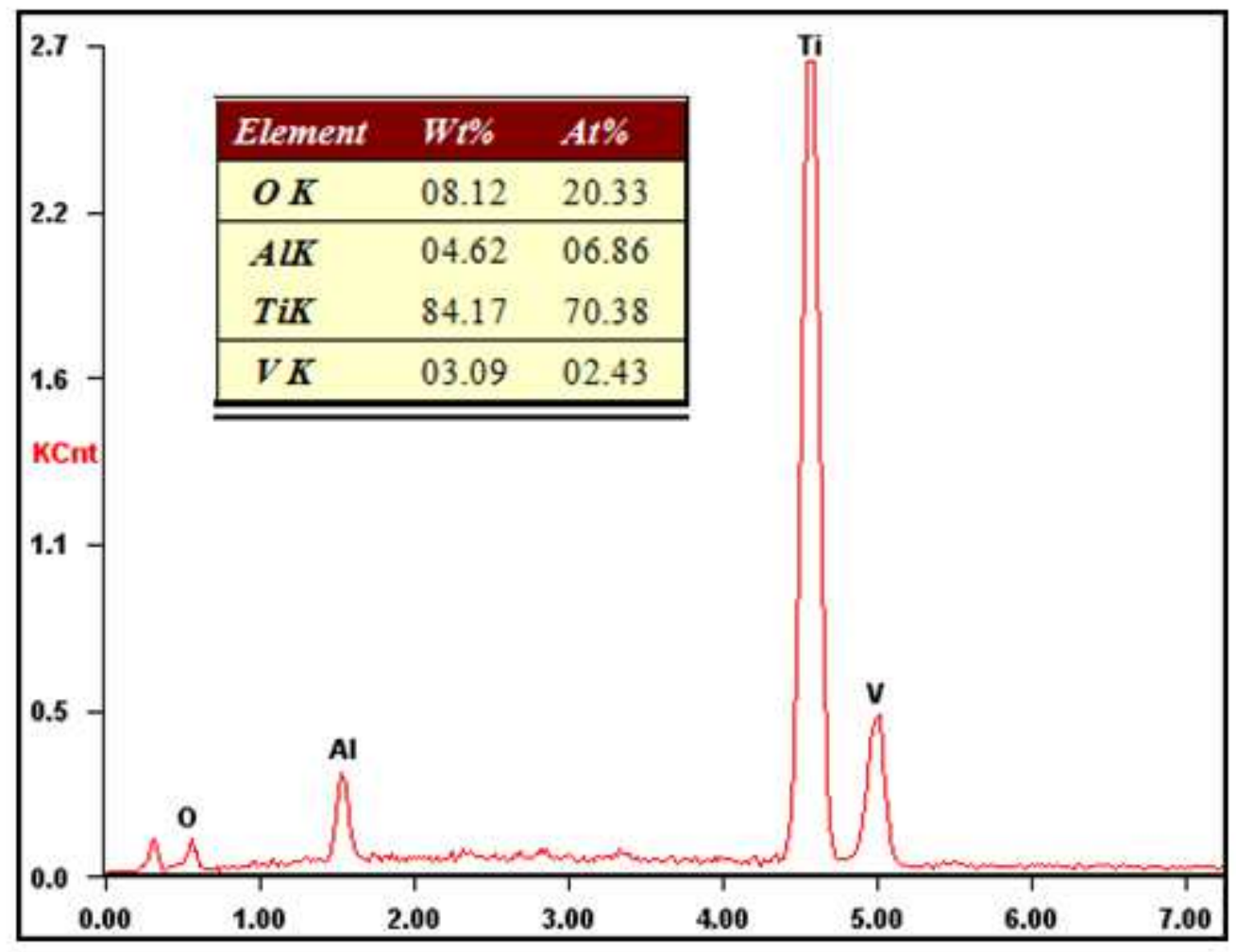

Figure 9

Energy spectrum analysis of the titanium alloy surface after EDM in water

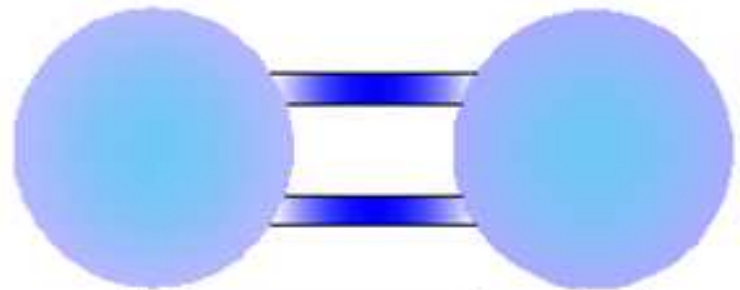

Oxygen molecule

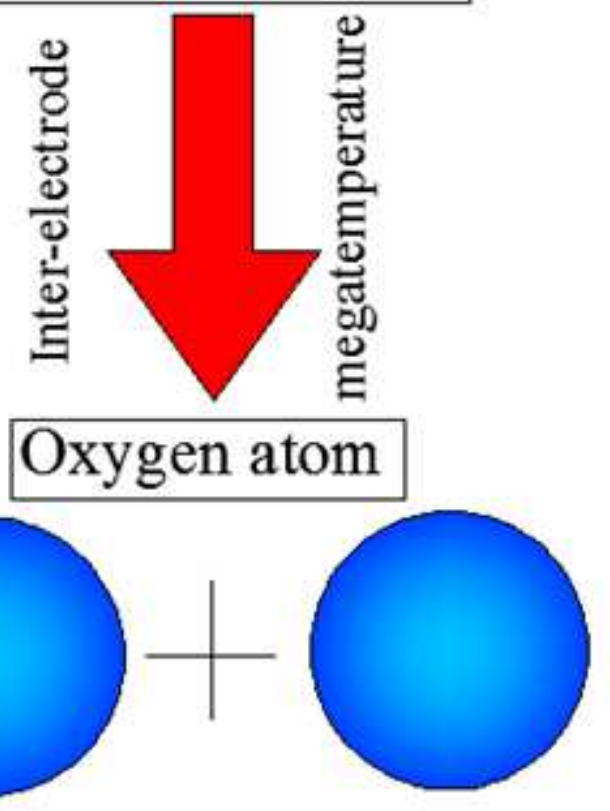


Figure 10

Dissociation of oxygen molecules.

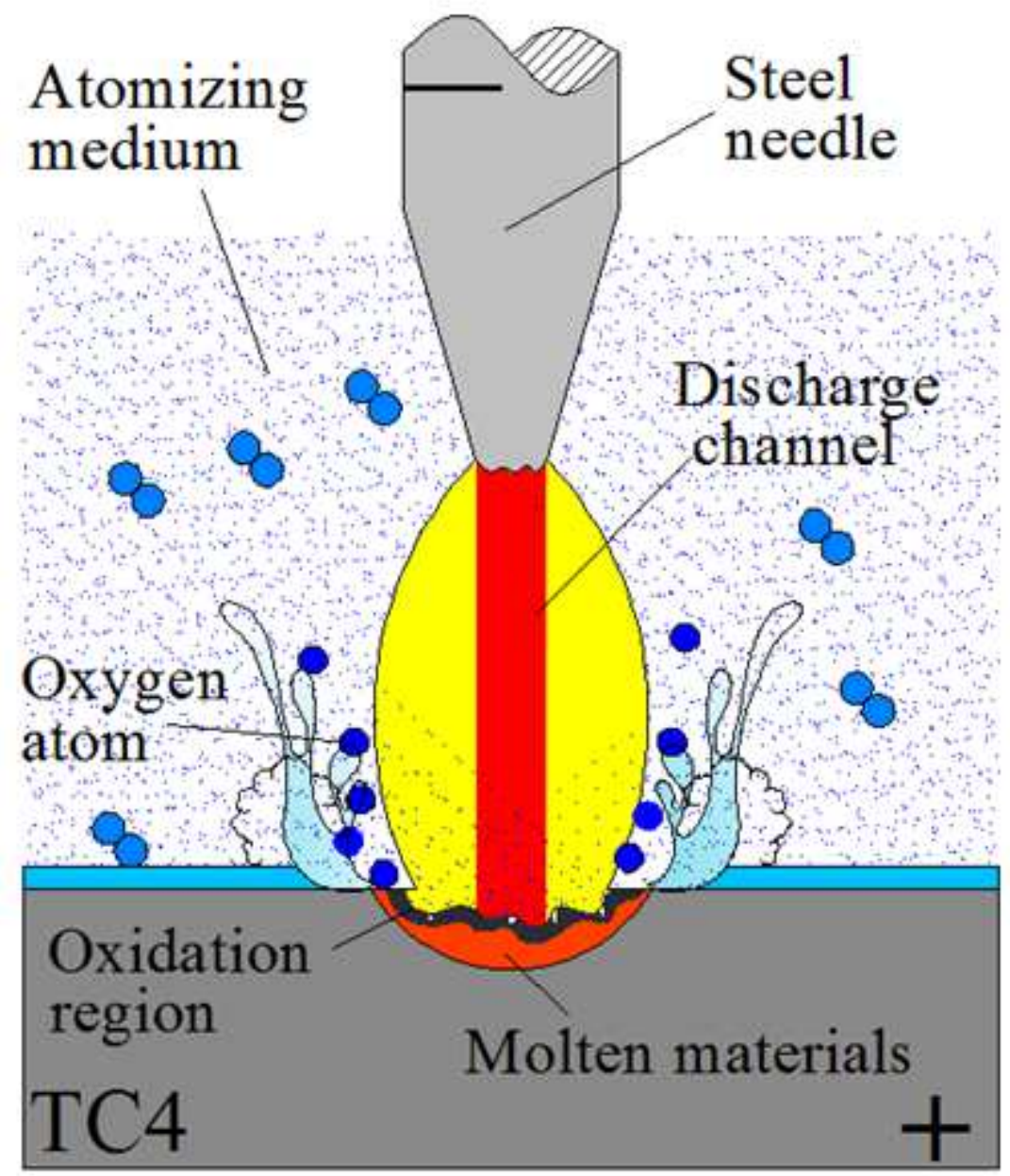

Figure 11

Oxidation occurrence and oxide layer generation 


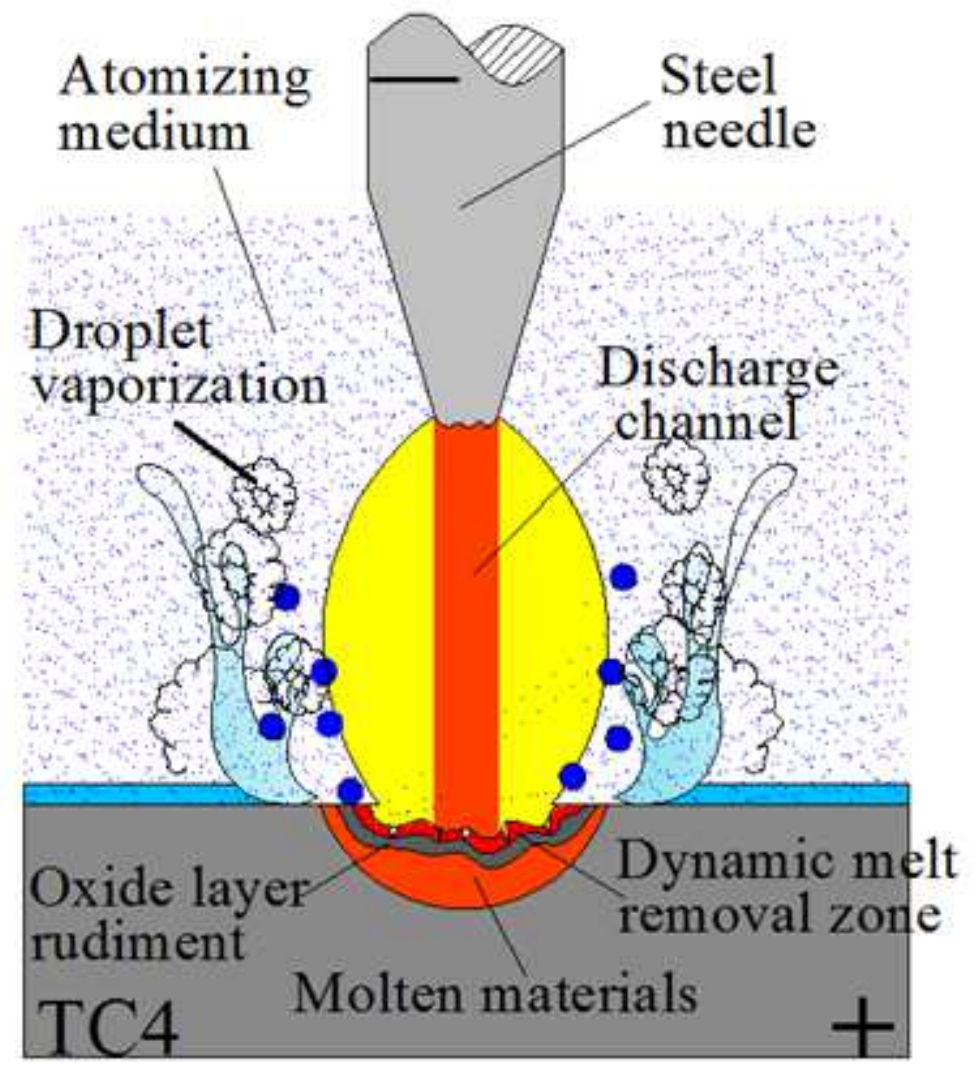

Figure 12

Oxidation-ablation dynamic balance and oxide layer prototype formation

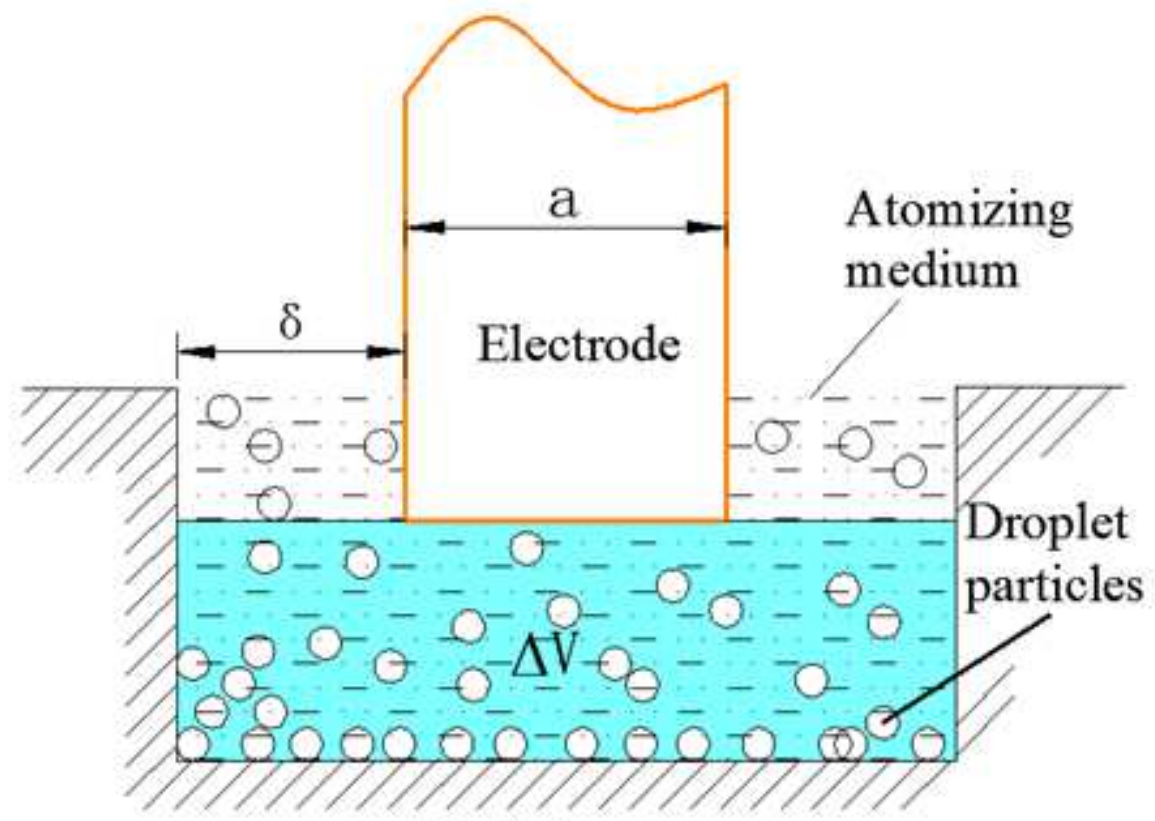

\section{Workpiece}

Figure 13

A model of inter-electrode atomization ablation 


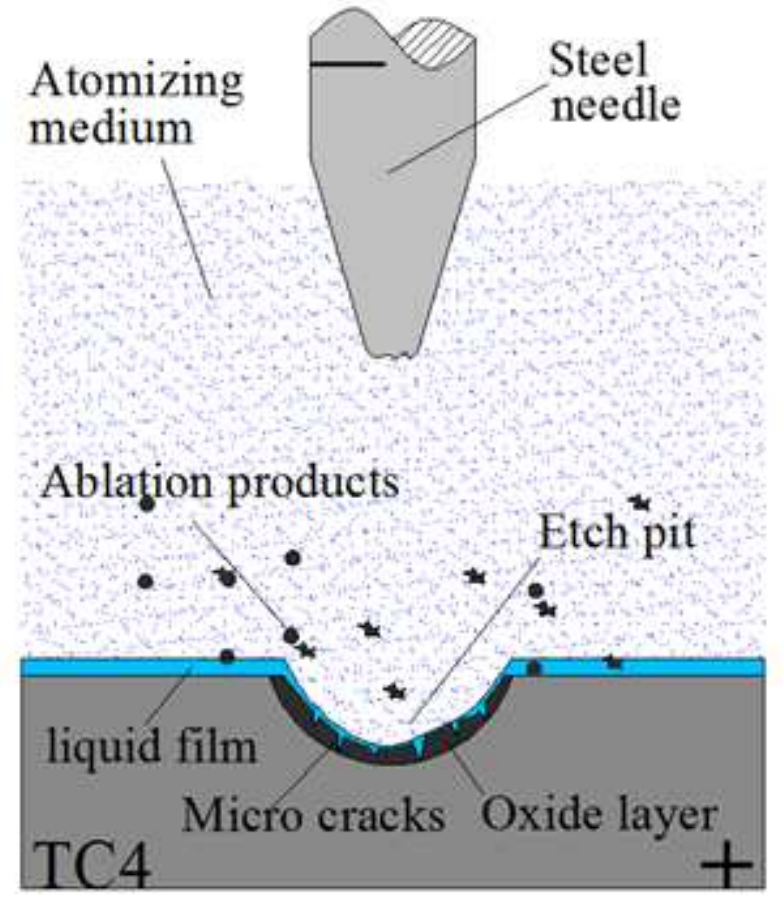

Figure 14

The end of discharge ablation and oxide layer formation.

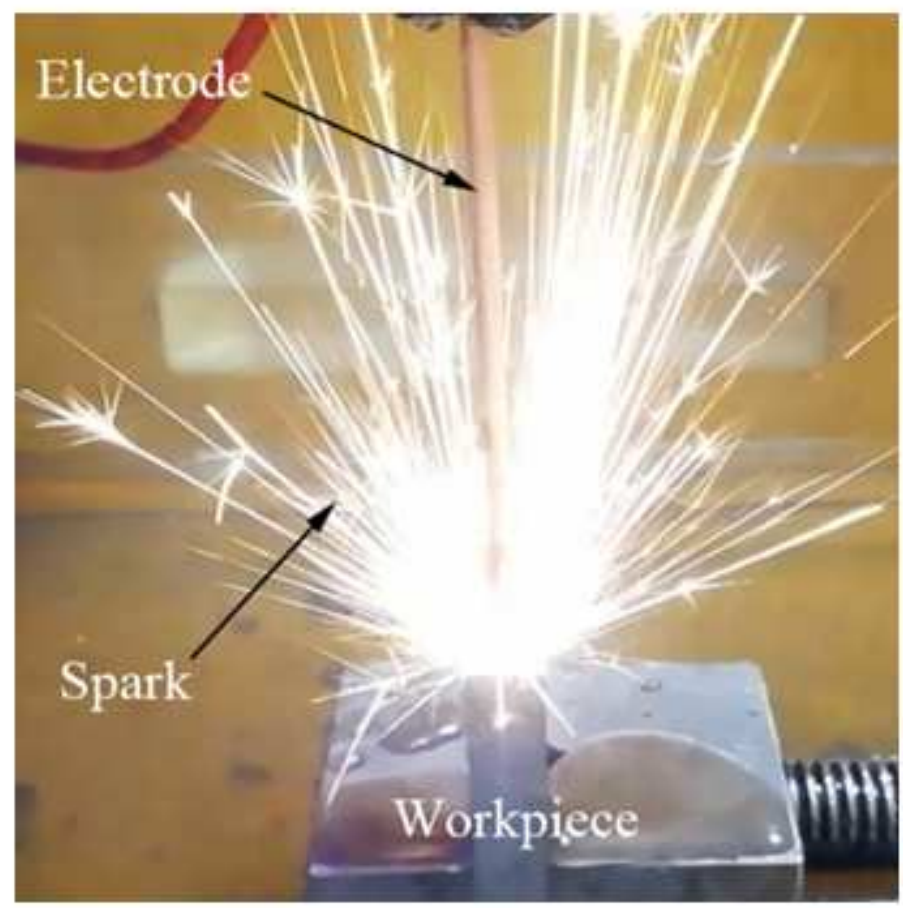

Figure 15

The MA-DAP machining status. 


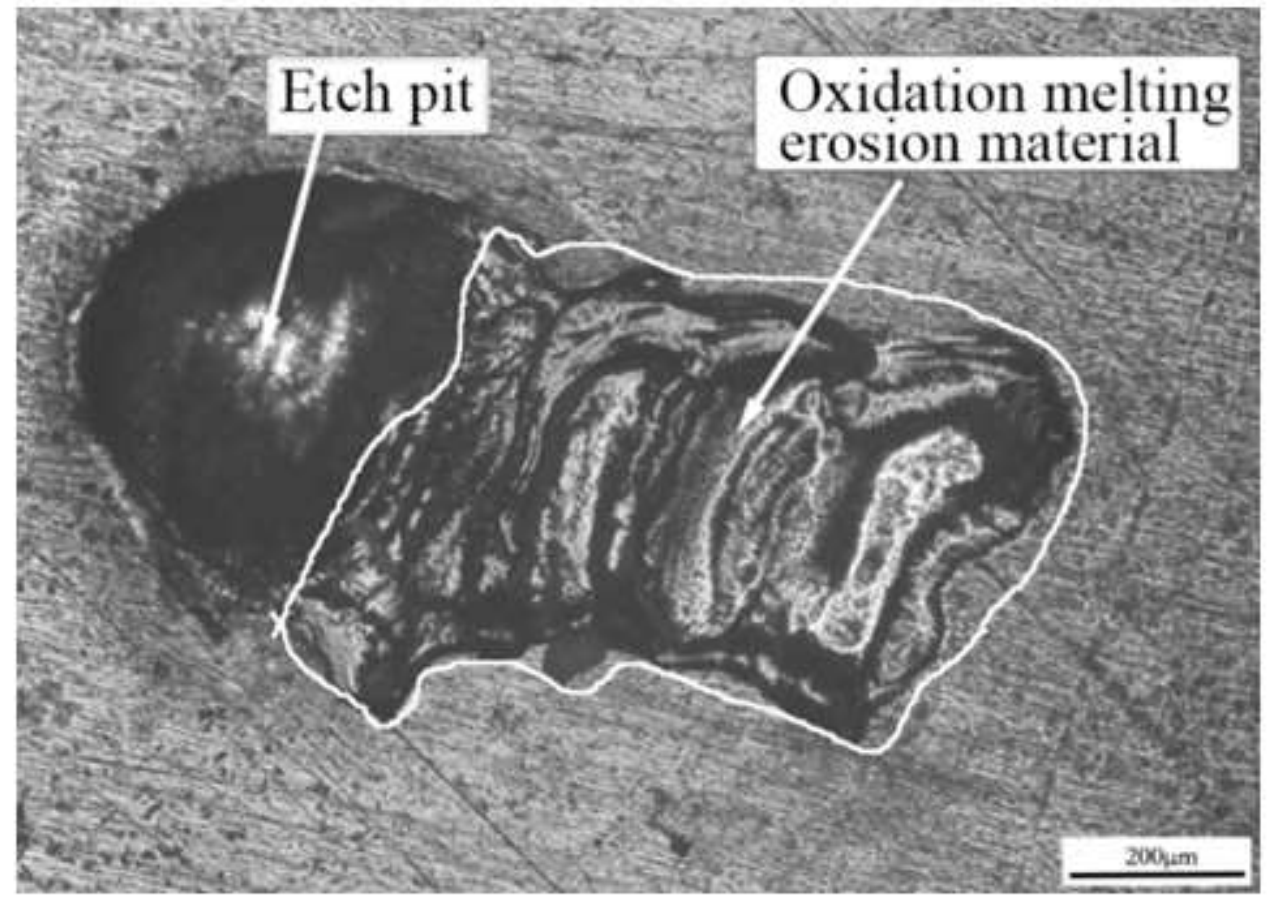

Figure 16

A discharge ablation pit.

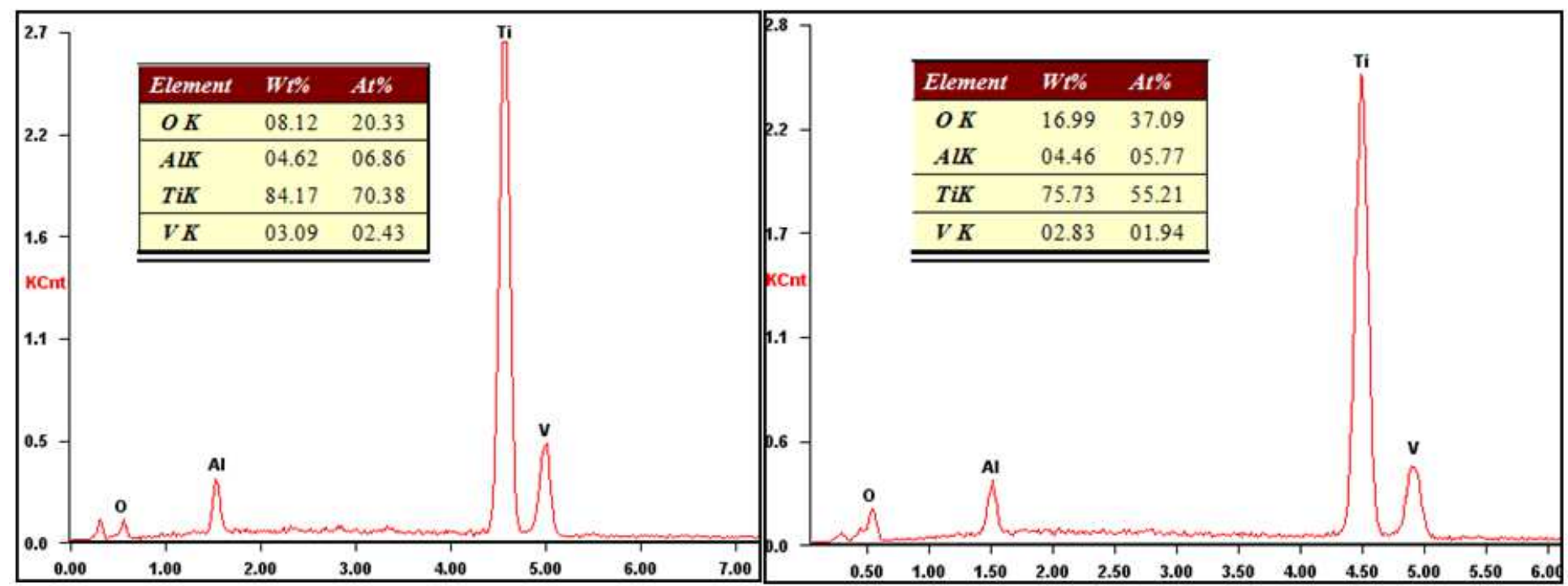

(a) Etch pit

(b) oxidation melting erosion material.

Figure 17

Discharge ablation pit surface energy spectrum detection 


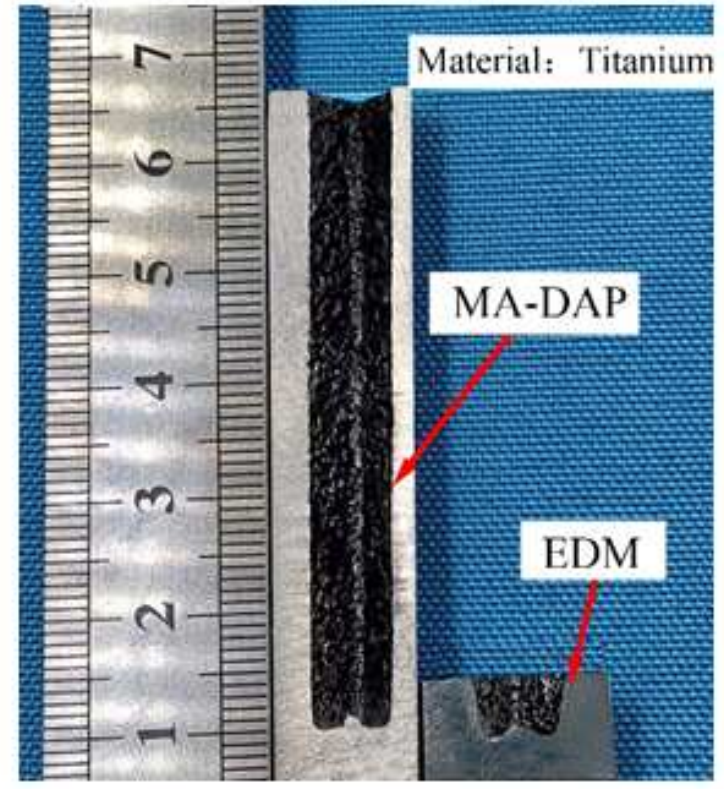

(a) Samples machined via EDM and MA-DAP

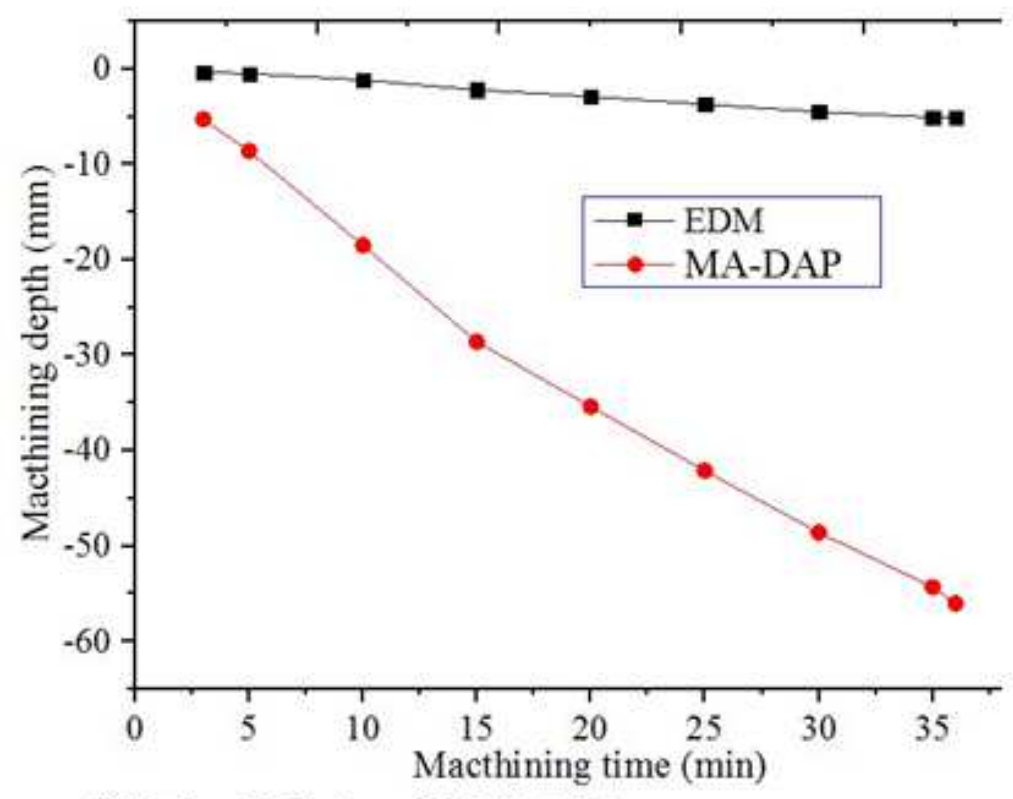

(b) The EDM and MA-DAP processes

Figure 18

EDM and MA-DAP comparative test results

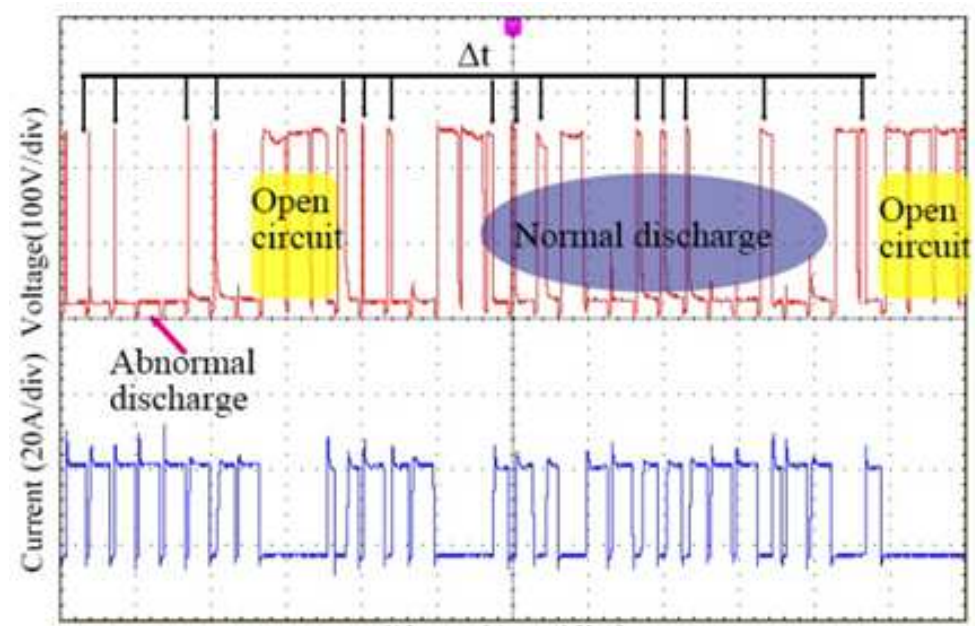

Time $(2 \mathrm{~ms} /$ div $)$

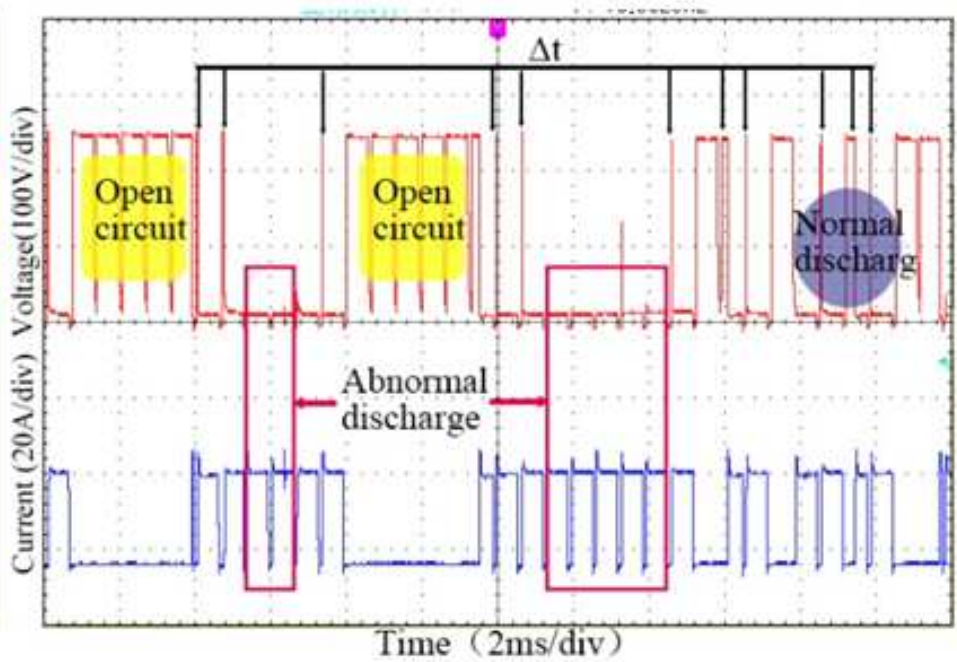

Time (2ms/div)

(a) MA-DAP (b) EDM

Figure 19

Comparison of discharge waveform 

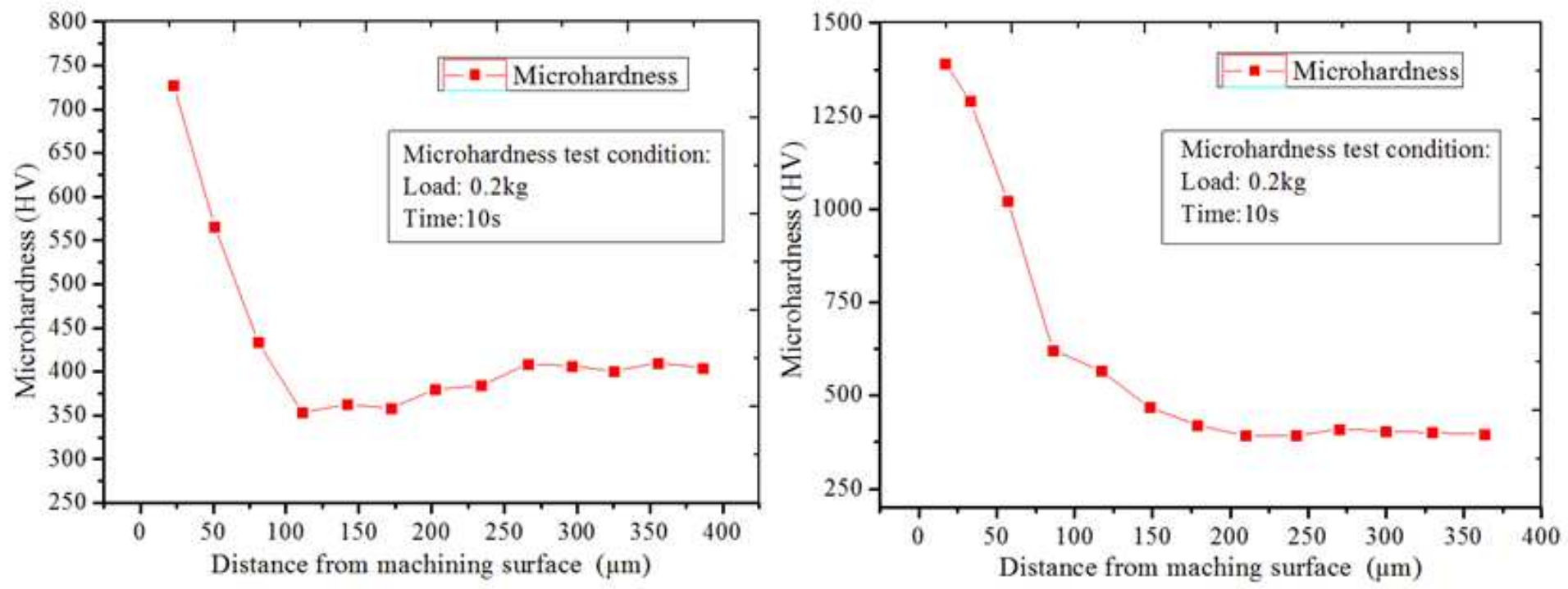

(a) MA-DAP (b) EDM

Figure 20

Cross-sectional hardness curves 